\title{
An Extension of the Time-Spectral Method to Overset Solvers
}

\author{
Joshua I. Leffell* \\ Stanford University, Stanford, CA 94305
}

\author{
Scott M. Murman ${ }^{\dagger}$ \\ NASA Ames Research Center, Moffett Field, CA 94035
}

Thomas H. Pulliam ${ }^{\ddagger}$

NASA Ames Research Center, Moffett Field, CA 94035

\begin{abstract}
Relative motion in the Cartesian or overset framework causes certain spatial nodes to move in and out of the physical domain as they are dynamically blanked by moving solid bodies. This poses a problem for the conventional Time-Spectral approach, which expands the solution at every spatial node into a Fourier series spanning the period of motion. The proposed extension to the Time-Spectral method treats unblanked nodes in the conventional manner but expands the solution at dynamically blanked nodes in a basis of barycentric rational polynomials spanning partitions of contiguously defined temporal intervals. Rational polynomials avoid Runge's phenomenon on the equidistant time samples of these sub-periodic intervals. Fourier- and rational polynomial-based differentiation operators are used in tandem to provide a consistent hybrid Time-Spectral overset scheme capable of handling relative motion. The hybrid scheme is tested with a linear model problem and implemented within NASA's OVERFLOW Reynolds-averaged NavierStokes (RANS) solver. The hybrid Time-Spectral solver is then applied to inviscid and turbulent RANS cases of plunging and pitching airfoils and compared to time-accurate and experimental data. A limiter was applied in the turbulent case to avoid undershoots in the undamped turbulent eddy viscosity while maintaining accuracy. The hybrid scheme matches the performance of the conventional Time-Spectral method and converges to the time-accurate results with increased temporal resolution.
\end{abstract}

\section{Introduction}

CTORCED periodic flows arise in a broad range of aerodynamic applications such as rotorcraft, turboma$\mathrm{F}$ chinery, and flapping wing configurations. Standard practice involves solving the unsteady flow equations forward in time until the initial transient exits the domain and a statistically stationary flow is achieved. It is often necessary to simulate through several periods of motion to remove the initial transient, making unsteady design optimization prohibitively expensive for most realistic problems. An effort to reduce the computational cost of these calculations led to the development of the Harmonic Balance method $[1,2]$ which capitalizes on the periodic nature of the solution. This approach exploits the fact that forced temporally periodic flow, while varying in the time domain, is invariant in the frequency domain. Expanding the temporal variation at each spatial node into a Fourier series transforms the unsteady governing equations into a coupled set of steady equations in integer harmonics that can be tackled with the acceleration techniques afforded to steady-state flow solvers. Other similar approaches, such as the Nonlinear Frequency Domain $[3,4,5]$, Reduced Frequency [6], and Time-Spectral [7,8,9] methods, were developed shortly thereafter. Additionally, adjoint-based optimization techniques [10,11] can be applied to provide the ability to perform design optimization without resorting to costly unsteady adjoint methods. Frequency-adaptive methods $[12,13,14]$ can offer even greater efficiency by refining the the number of modes resolved at each grid point to the frequency content in its solution.

\footnotetext{
*Department of Aeronautics \& Astronautics, AIAA Student Member. jleffell@stanford.edu

${ }^{\dagger}$ AIAA Member. Scott.M.Murman@nasa.gov

¥AIAA Associate Fellow. Thomas.H.Pulliam@nasa.gov
} 
The Fourier temporal basis functions imply spectral convergence as the number of harmonic modes, and correspondingly, the number of time samples, $N$, increases. Some approaches solve the equations in the frequency domain directly. Others transform the equations back into the time domain, potentially simplifying the process of augmenting this capability to existing solvers. However, each approach harnesses the underlying steady solution in the frequency domain. These temporal projection methods will herein be collectively referred to as Time-Spectral methods.

Time-Spectral methods have demonstrated marked success in reducing the computational costs associated with simulating periodic forced flows, but have yet to be generally applied to overset or Cartesian solvers for relative motion with dynamic hole-cutting. Overset and Cartesian grid methodologies are versatile techniques capable of handling complex geometry configurations with relative motion between components, and are commonly used for practical engineering applications. The combination of the Time-Spectral approach with this general capability potentially provides an enabling new design and analysis tool. In an arbitrary moving-body scenario for these approaches, a Lagrangian body-fixed mesh moves through a fixed Eulerian mesh. Eulerian mesh points interior to the solid body are removed (cut or blanked), leaving a hole in the Eulerian mesh. These grid points undergo dynamic blanking and do not have a complete set of time samples, preventing direct application of the Time-Spectral approach. Murman [6] demonstrated the Time-Spectral method for a Cartesian solver with rigid domain motion, wherein the hole cutting remained fixed. Similarly, Custer et al. $[15,16]$ used the NASA overset OVERFLOW solver with static hole-cutting and interpolation. Both of these efforts focused on applying this method to Cartesian and overset meshes with constant blanking. Recently, Mavriplis et al. [17] demonstrated a qualitative method for applying the Time-Spectral approach to an unstructured overset solver with relative motion.

The goal of the current work is to develop a robust and consistent method for handling relative motion with the Time-Spectral approach within an overset or Cartesian mesh framework, while still approaching the spectral convergence rate of the non-overset scheme. The paper begins in Section II with a brief outline of the Time-Spectral approach and the issues associated with extending it to overset solvers. Section III details the proposed method that enables relative-body motion and provides convergence properties of the new differentiation operator. A linear model problem demonstrates the viability of the approach. Section IV outlines the implementation of the approach within OVERFLOW. The paper concludes with application of the Time-Spectral OVERFLOW solver to two-dimensional simulations of both plunging and pitching airfoils to evaluate the accuracy and convergence properties of the proposed scheme.

\section{Application of the Time-Spectral Method within an Overset Framework}

The fundamental challenge of providing Time-Spectral capabilities to an overset solver concerns the nodes that dynamically move in and out of the physical domain due to the relative motion between the surface geometry and the background Eulerian grid(s). Projection into the frequency domain has infinite support in time, requiring special treatment for these nodes whose solution is undefined for a subset of the temporal period. Figure 1 illustrates this issue for a one-dimensional oscillating piston. Fictional solutions in Fig. $1 \mathrm{~b}$ corresponding to nodes $a, b$ and $c$ in Fig. 1a demonstrate dynamic hole cutting by the motion of the piston relative to the background Eulerian grid. Node $a$ is never blanked by the piston and therefore its solution is defined over the entire time history of the period, $T$. The piston blanks node $b$ briefly and node $c$ twice. Thus, node $b$ has one sub-periodic time interval associated with it while node $c$ has two - one each represented by the solid and hashed lines. The shaded regions serve to highlight the time over which each node lies outside the physical domain with an undefined solution. Special treatment is required for nodes $b$ and $c$, while the standard Time-Spectral approach can be applied to node $a$, resulting in a hybrid scheme.

For a brief synopsis of the standard Time-Spectral approach, consider a time-dependent scalar ODE

$$
\frac{\partial u}{\partial t}+R(u)=0 .
$$

The continuous solution is expanded in the harmonic basis

$$
u(t)=\sum_{k=0}^{N-1} \hat{u}_{k} \phi_{k}(t)
$$

from data at equispaced time samples, $u_{j}=u\left(t_{j}\right)=u(j \Delta t)$, where $\Delta t=\frac{2 \pi}{\omega N}$, such that

$$
\hat{\mathbf{u}}=\Phi^{-1} \mathbf{u}
$$




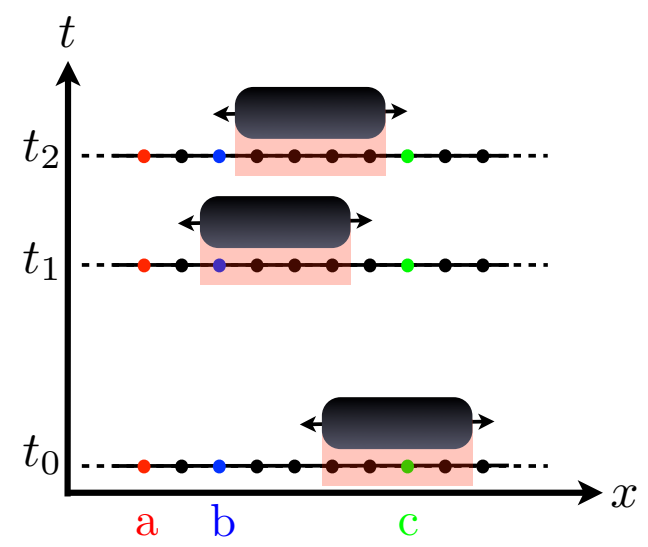

(a) Piston Motion

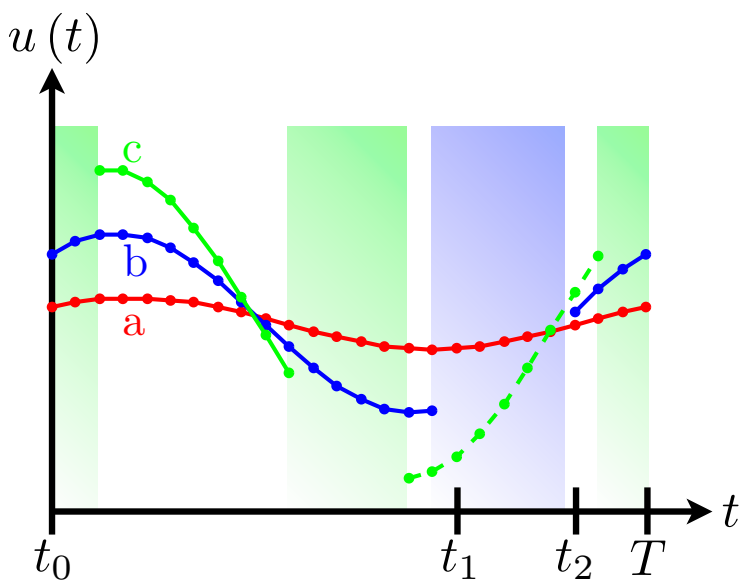

(b) Solution Time Histories

Figure 1: Figurative piston trajectory and solution histories. (a) The piston "cuts" nodes $b$ and $c$ as it oscillates over the background grid. (b) Shaded regions represent blanked intervals through which the solution is undefined for nodes of the corresponding color.

with

$$
\Phi_{j k}=\phi_{k}\left(t_{j}\right)=e^{i \omega k t_{j}}
$$

serving as the transformation operator. The differentiation operator is applied in the frequency domain, i.e. $\frac{d}{d t} \hat{u}_{k}=i \omega k \hat{u}_{k}$, and the result is projected back into the time domain leading to

$$
\Phi D \Phi^{-1} \mathbf{u}+\mathbf{R}(\mathbf{u})=0
$$

where $D$ is the diagonal Fourier differentiation operator, $d_{j k}=i \omega k \delta_{j k}$. Direct assembly of the transformed differentiation operator, $\mathcal{D}=\Phi D \Phi^{-1}$, is derived in $[7,8]$. Iterating Eq. 5 in pseudotime, $\tau$, provides a steady-state solution procedure to an underlying unsteady, yet periodic, problem. ${ }^{\text {a }}$

$$
\frac{d}{d \tau} \mathbf{u}+\mathcal{D} \mathbf{u}+\mathbf{R}(\mathbf{u})=0
$$

The majority of spatial nodes in an overset or Cartesian simulation possess complete time histories and are thus treated with this standard procedure, but an accurate and robust treatment is still required for the complement of nodes that undergo dynamic blanking.

One approach is to fill the blanked nodes with solution data via spatial averaging and proceed with the standard Time-Spectral method (cf. [17]). While attractive for its simplicity, this approach is inconsistent. Non-physical information provided by an alternative governing equation (spatial smoothing is governed by Laplace's equation) is propagated into the physical domain via the infinite support of the Fourier basis. To demonstrate this inconsistency, see Fig. 2 which shows the solution of the one-dimensional linear advection equation

$$
\frac{\partial u}{\partial t}+a \frac{\partial u}{\partial x}=0
$$

given $u(0, t)=-\sin (\omega a t)$ for $x \in[0,1]$. The analytic solution is $u(x, t)=\sin (\omega(x-a t))$. A gap is defined in $x$ to simulate blanking and the solution is linearly interpolated through the gap at every step. The interpolation error across the spatial gap (Fig. 2a) at one time-sample corrupts the solution throughout the

\footnotetext{
${ }^{a}$ Alternatively, the residual operator can also be projected into the frequency domain, and the resulting equations solved iteratively in the frequency domain [3,6]. This approach takes advantage of the Fast Fourier Transform which is an $O(N \log N)$ operation as opposed to the $O\left(N^{2}\right)$ matrix operation in Eq. 5. However, for the small $N$ typically encountered in practical 3D Time-Spectral applications $(O(10-100))$ the difference is minimal and the time domain formulation allows for straightforward implementation within a steady-state solver with the addition of the temporal differentiation source term.
} 
period (Fig. 2b) via the infinite support of the Fourier basis. While the resulting solution may be smooth, it is inaccurate and dependent upon the arbitrary averaging of the solution through the gap. Consequently, spatial interpolation inhibits the desired temporal convergence (Fig. 2c). If instead the node located at $x=0.5$ were blanked, the error in the solution would be much lower because the solution at this location can be more accurately approximated by linear interpolation between its neighbors.

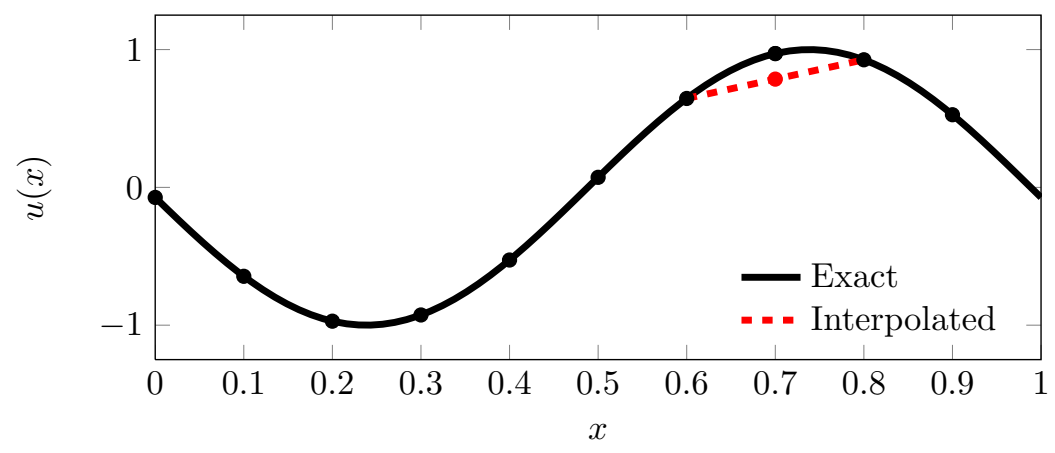

(a) "Geometry" gap and interpolation region

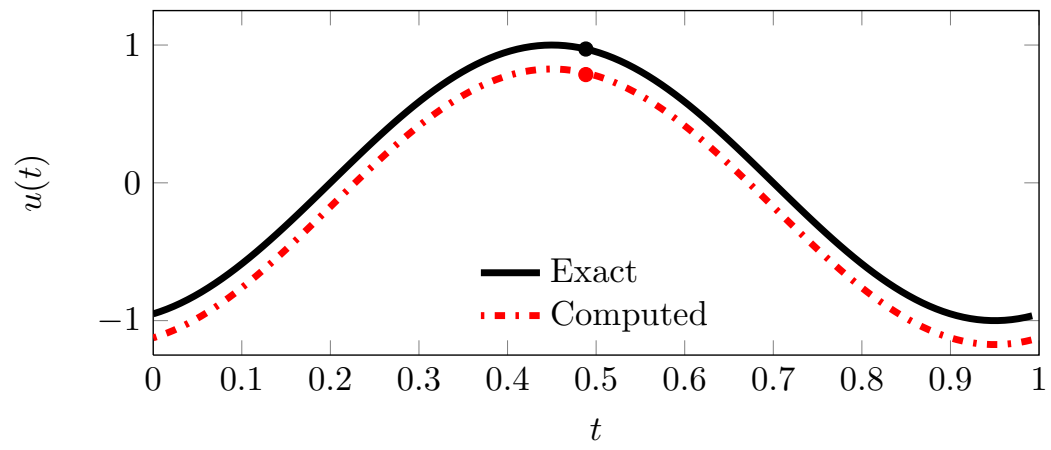

(b) Exact and computed solutions.

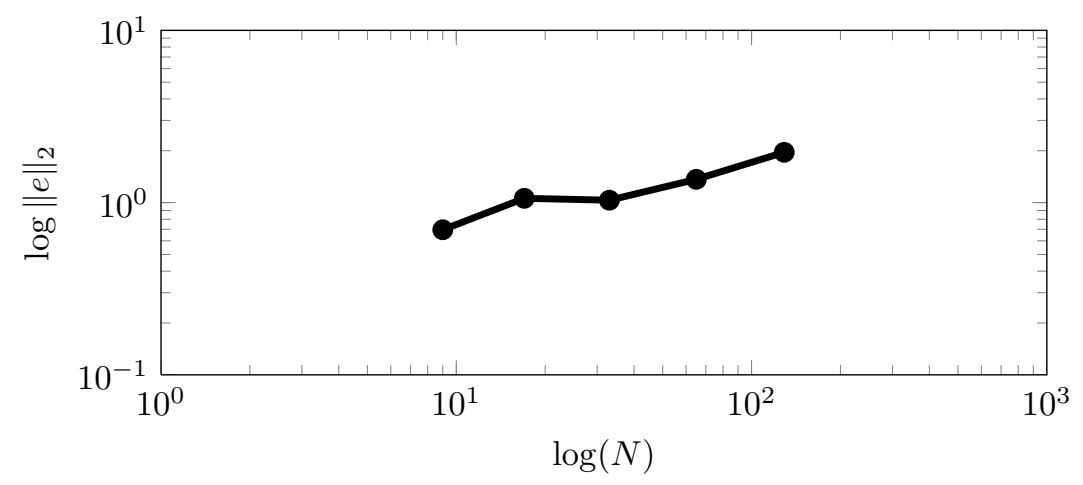

(c) Convergence of analytic error, $e=\left|u-u_{e x}\right|$, with $N$

Figure 2: Spatial smoothing Time-Spectral approach with a blanked node at $x=0.7$ (a) The solution at the blanked node is interpolated from its neighbors. (b) The solution resulting from spatial averaging may be smooth but is inconsistent, as the arbitrarily filled value at the blanked node corrupts its complete time history and, by extension, the entire space-time domain. Severity of the corruption may be masked by fortuitous interpolation but it is highly dependent on the underlying solution and the gap through which it is filled. (c) Spatial averaging of the blanked point introduces an error that inhibits temporal convergence as the number of temporal modes, $N$, is increased. 


\section{Hybrid Fourier-Rational Time-Spectral Approach}

To develop a consistent scheme without the issues associated with spatial averaging presented in the previous section, the current approach partitions the time histories for spatial nodes exhibiting dynamic blanking into intervals of consecutively unblanked time samples. Global temporal support is thus abandoned, however, this approach is consistent with the physics of disjoint domains separated by an impermeable boundary. It is important to note that these temporal partitions must still be evaluated on a uniform lattice to avoid manipulating the spatial operator. Thus the problem is now reduced to finding a robust and efficient method for evaluating the temporal derivatives of non-periodic functions on a uniform lattice.

Several approaches to achieve this goal were evaluated. One option is to compute derivatives with finite differences or a localized differentiable basis such as wavelets. However, compact bases offer limited accuracy [18] and wavelet differentiation requires special treatment at non-periodic interval boundaries [19]. Another option is temporal extrapolation that populates the undefined portion of the period using data from the physical portion of the time signal. Examples of this approach, such as Fourier continuation, which extrapolates a non-periodic function into a periodic function on a larger domain [20,21,22], and compressed sampling, which requires $L_{1}$ minimization to solve an underdetermined system [23], are both too costly and lack the requisite robustness for this application.

The current approach projects the solution of the unblanked nodes within a single partition onto a global basis spanning the same interval. The constraint of evenly-spaced temporal collocation points eliminates traditional optimally-accurate orthogonal polynomials (e.g. Chebyshev) due to Runge's phenomenon at the endpoints. A least-squares projection of orthogonal polynomials is more stable, but results in an overdetermined system whose projection does not interpolate the solution data. Barycentric rational polynomials provide a viable alternative [24] and Bos et al. [26] showed that they offer superior interpolation and differentiation properties on equidistant nodes over conventional orthogonal polynomials. A rational polynomial-based differentiation operator will therefore be used in this hybrid Time-Spectral approach. An alternate basis may prove more successful in the future so the current approach will be kept general enough to use any projection operator, contingent on the availability of its analytic differentiation operator. In the proposed scheme, solutions across each non-periodic interval are projected onto a basis of rational polynomials and differentiated accordingly. Fully periodic intervals are still projected and differentiated in the Fourier basis, resulting in a hybrid approach that employs the optimal basis available.

Floater and Hormann [25] introduced a set of barycentric rational polynomials with high rates of approximation convergence and no poles. Additional efforts to explore rational polynomials and their utility as a pseudospectral basis for spectral collocation methods include but are not limited to $[27,28,29,30]$. Baltensperger et al. [31] define the rational interpolant, $r(x)$, in barycentric form

$$
r(x)=\frac{\sum_{k=0}^{N} \frac{w_{k}}{x-x_{k}} f_{k}}{\sum_{k=0}^{N} \frac{w_{k}}{x-x_{k}}}
$$

and its corresponding differentiation operator, $D$, such that $\frac{d}{d x} \mathbf{r}=D \mathbf{r}$

$$
D_{j k}= \begin{cases}\frac{w_{k}}{w_{j}} \frac{1}{\left(x_{j}-x_{k}\right)} & \text { if } j \neq k \\ -\sum_{i=0, i \neq k}^{N} D_{j i} & \text { if } j=k\end{cases}
$$

where $r_{k}=r\left(x_{k}\right)$. The interpolation can be reformulated as a weighted sum of nodal basis functions, $\phi(x)$, with coefficients, $\hat{f}$, equal to the function value at each node, $\hat{f}_{k}=f\left(x_{k}\right)$.

$$
f(x)=\sum_{k=0}^{N} \hat{f}_{k} \phi_{k}(x), \quad \text { with } \phi_{k}(x)=\frac{\frac{w_{k}}{x-x_{k}}}{\sum_{j=0}^{N} \frac{w_{j}}{x-x_{j}}}
$$

Barycentric rational polynomials nodally interpolate the solution data resulting in an identity transformation operator, $\Phi_{j k}=\phi_{k}\left(x_{j}\right)=\delta_{j k}$, implying discrete orthogonality. It follows that the transformed differentiation 
operator is identical to $D: \mathcal{D}=\Phi D \Phi^{-1}=D$. While similar in form to the Lagrange interpolant, a key distinction of the barycentric rational polynomials is in how the weights, $w_{k}$, are defined. Lagrange polynomials are constrained to pass through $N+1$ points as a polynomial of order $N$. In contrast, while the rational polynomials interpolate the data, they are not constrained to do so as a polynomial of order $N$. This relaxation aids in preventing spurious oscillations at the endpoints of equispaced nodes associated with the traditional Lagrange or Chebyshev polynomial bases while retaining powerful interpolation and differentiation properties. The weights, $w_{k}$, control the accuracy and stability characteristics of the rational interpolant. Floater and Hormann [25] derived weights that provide an approximation order of $d$ while avoiding poles. Weights guaranteeing an absence of poles

$$
w_{k}=(-1)^{k-d} \sum_{i \in J_{k}} \prod_{j=i, j \neq k}^{i+d} \frac{1}{\left|x_{k}-x_{j}\right|}
$$

are defined for $N+1$ samples where

$$
I_{T}:=\{0,1, \ldots, N-d\} \text { and } J_{\alpha}:=\left\{i \in I_{T}: \alpha-d \leq i \leq \alpha\right\}
$$

for the desired approximation order $d$. Readers are directed to the derivation in [25] for additional details. Figure 3 illustrates three basis functions corresponding to the first, second and fourth nodes with approximation order, $d=3$, over 8 sample points $(N=7)$ with the values highlighted at the nodal points. Note the interpolation property of the basis, $\phi_{k}\left(x_{j}\right)=\delta_{j k}$.

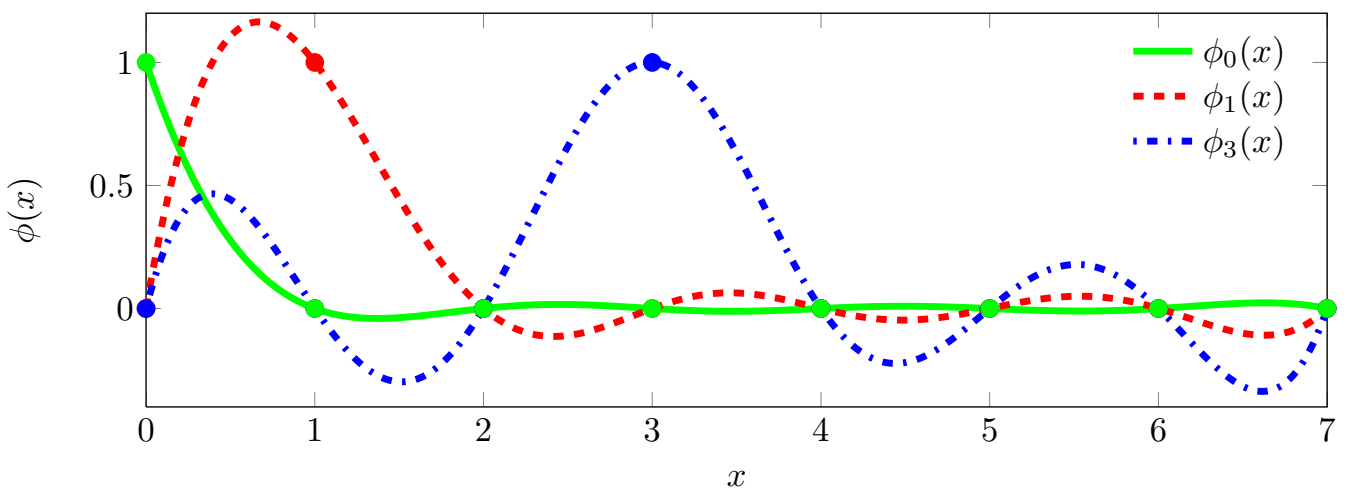

Figure 3: Barycentric rational polynomial basis functions $\phi_{0}(x), \phi_{1}(x)$ and $\phi_{3}(x)$ corresponding to nodes 0 , 1 and 3, respectively, for approximation order, $d=3$, and 8 equispaced sample points.

Differentiation performance of the rational polynomial basis is compared to that of third-order finitedifferences, the Fourier differential operator (generally optimal for periodic functions), differentiation using least-squares orthogonal polynomials on evenly-spaced nodes (modes $M \leq 2 \sqrt{N}$ ) and the Chebyshev differential operator (generally optimal for non-periodic functions) on Chebyshev nodes. An even-odd harmonic function and Runge's function (designed to expose numerical instabilities) are differentiated by each of the aforementioned methods. Convergence of differentiation error, $e=\left\|\frac{d f}{d x}-\mathcal{D} f\right\|_{2}$, with $N$ is plotted in Fig. 4 for the harmonic function and Fig. 5 for Runge's function. The approximation order for the rationals is selected as $d=\min \left(\left\lfloor\frac{N}{2}\right\rfloor, d_{\max }\right)$ with $d_{\max }=10$ for this case, but optimal selection is problem dependent and an area of continuing research $[29,32]$. The rational polynomials approach spectral convergence for both the periodic and non-periodic problems. Fourier differentiation exhibits roundoff error for the harmonic function with increased $N$ and offers poor convergence for Runge's function. 


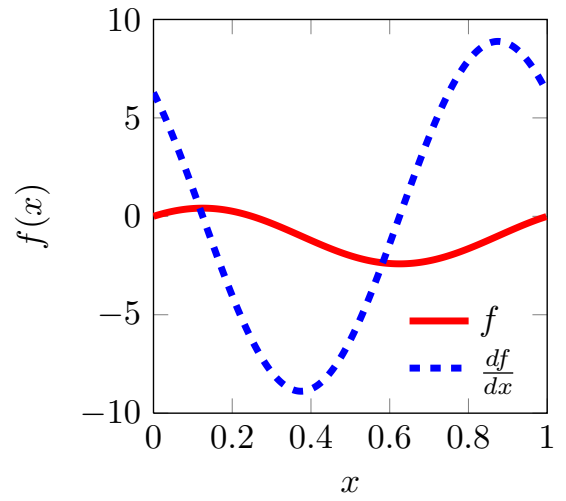

(a) Analytic function and derivative

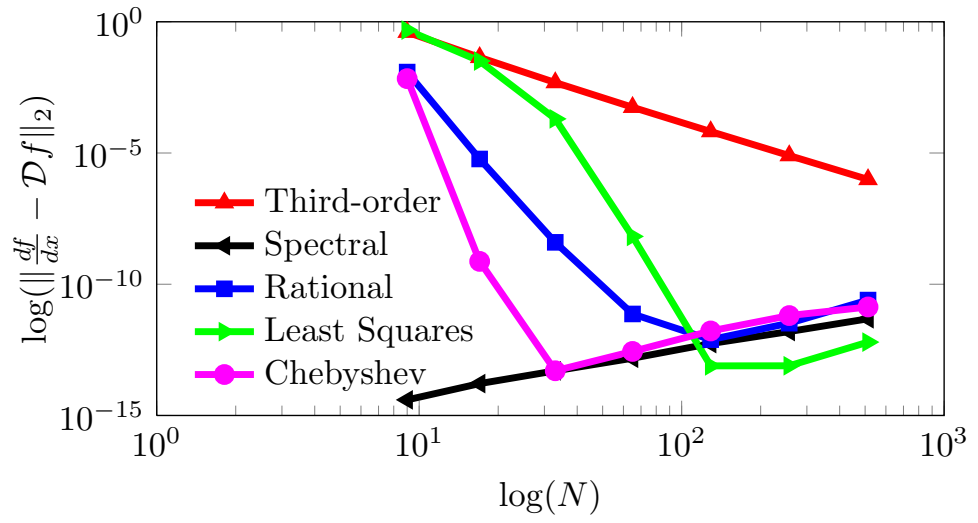

(b) Convergence of differentiation operators

Figure 4: Convergence of differentiated harmonic function, $f(x)=\cos (\omega x)+\sin (\omega x)$

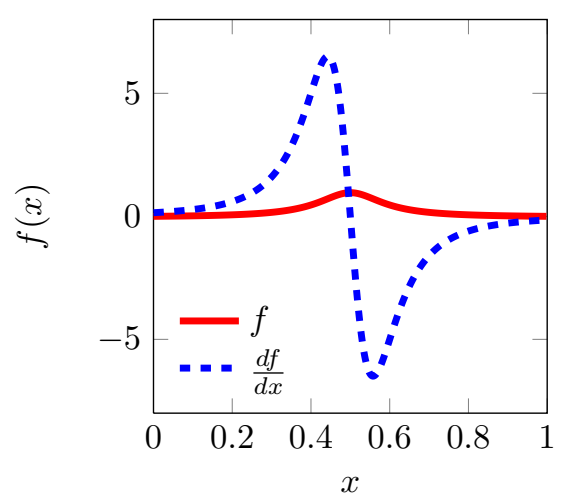

(a) Analytic function and derivative

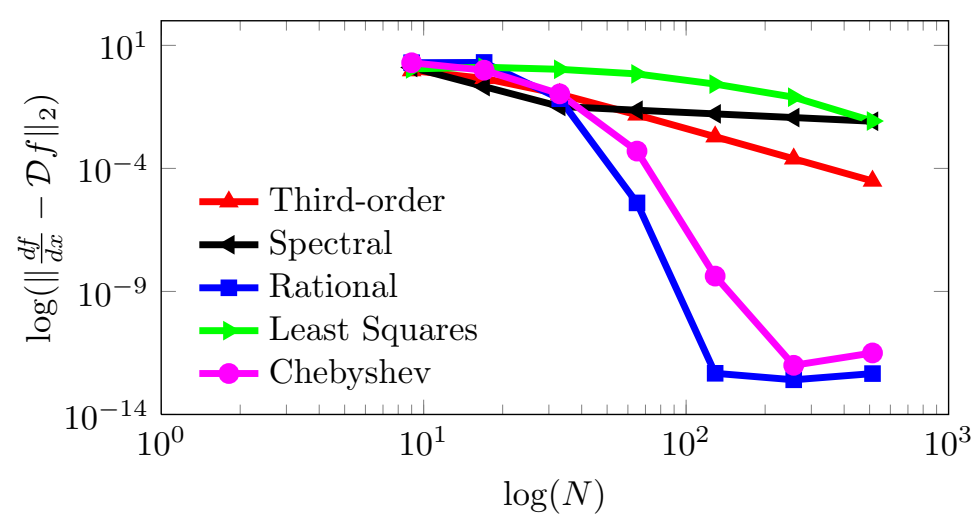

(b) Convergence of differentiation operators

Figure 5: Convergence of differentiated Runge's function, $f(x)=\frac{1}{1+25 x^{2}}$

To demonstrate the viability of the proposed approach, the same one-dimensional linear advection case (Eq. 7) shown in Sec. II is used to test the hybrid Time-Spectral strategy. Instead of the spatial interpolation used previously, the barycentric rational polynomial temporal differentiation operator, $\mathcal{D}_{r}$, is used on the spatial nodes blanked over some duration of the period and the Fourier differentiation operator, $\mathcal{D}_{f}$, is used on nodes with a periodic interval. Information propagates from left to right with wave speed $a=1$ and thus analytic boundary conditions are supplied both at $x=0$ and along the right edge of the blanked region. The domain is discretized with $N_{x}=101$ points in space and $N=21$ time samples. The solution is initialized uniformly to zero outside the boundary nodes, and third-order finite-differences are used to approximate spatial derivatives to minimize errors associated with the spatial discretization. The equations are solved implicitly and converged to machine precision. The space-time solution, visualized in Fig. 6, is compared to the analytic solution and the solution of the unblanked case. 


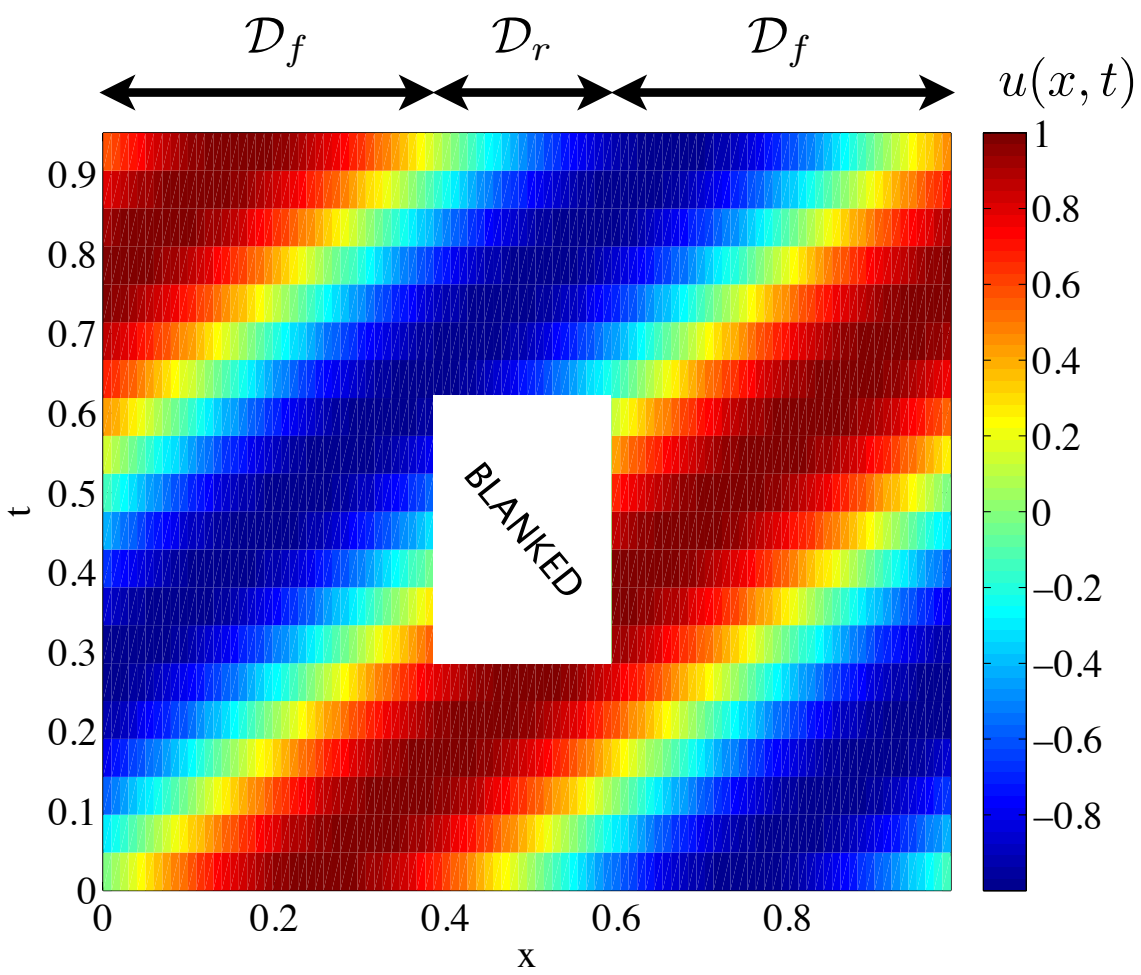

(a) Space-time solution

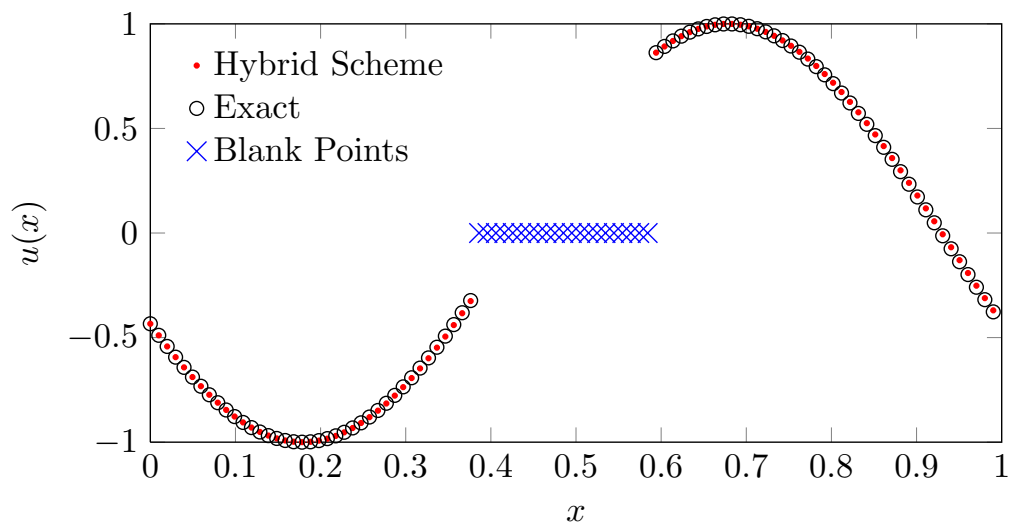

(b) Solution and blanked interval at fixed time $t=0.4286$

Figure 6: Solution for linear advection test problem using the hybrid Fourier-rational polynomial TimeSpectral approach. The rational polynomial differentiation operator, $\mathcal{D}_{r}$, is applied to nodes in the central region of the domain that undergo blanking and the Fourier differentiation operator, $\mathcal{D}_{f}$, is applied elsewhere.

The maximum error between the solution computed with the hybrid scheme and the analytic solution is $7.757 \times 10^{-3}$. This value is just slightly larger than the maximum error between the unblanked case using only the Fourier differentiation operator and the analytic solution, $7.717 \times 10^{-3}$. The largest difference between the two solutions is $5.485 \times 10^{-3}$ suggesting that the Fourier-rational hybrid approach essentially produces the same result as the conventional Time-Spectral method in the active region. 


\section{Implementation of the Hybrid Time-Spectral Approach in OVERFLOW}

The Time-Spectral approach requires no modification to the spatial operator, making the scheme straightforward to implement and maintain within an existing code. The current work leverages the OVERFLOW overset flow solver [33], which provides a mature code base for solving the three-dimensional compressible Reynolds-averaged Navier-Stokes (RANS) equations using a structured finite-difference scheme. OVERFLOW employs an approximate-factorization algorithm for the implicit solution of the spatial derivatives, and the Time-Spectral operator is easily integrated within this framework by the addition of a similar factored operator for the time derivative. This approximate factorization avoids forming and solving a $N_{x}^{3} \times N_{Q} \times N$ system of equations coupled across time and space. Writing the full Time-Spectral approximate-factorization difference equation in "delta" form gives

$$
\left[I+\Delta \tau A_{x}\right]\left[I+\Delta \tau A_{y}\right]\left[I+\Delta \tau A_{z}\right][I+\Delta \tau \mathcal{D}] \Delta \vec{Q}=-\Delta \tau\left[R\left(\vec{Q}^{n}\right)+S\left(\vec{Q}^{n}\right)\right]
$$

where $R\left(\overrightarrow{Q^{n}}\right)$ is the standard nonlinear spatial residual operator, and $S\left(\overrightarrow{Q^{n}}\right)$ is the Time-Spectral differential operator applied to the state vector at time level $n . A_{x}, A_{y}$ and $A_{z}$ are the linearized spatial operators corresponding to the $x-, y$ - and $z$-directions. The Time-Spectral approach as applied to OVERFLOW avoids modifying the existing spatial residual and implicit operators because they are applied to each time sample sequentially within each iteration. The required modifications are limited to an additional linear solve of dimension $N$ for the Time-Spectral factored operator, and computation of the source term at every spatial grid point. In other words, time is treated as an additional spatial dimension when solving for the steady-state solution in the combined space-time domain.

The computational memory requirements for a Time-Spectral solution are greater than its time-accurate analog because the solution and source term must be stored for each time sample. However, careful implementation limits the storage requirements and allows for a significant number of temporal modes even in complex three-dimensional problems. Ignoring the trivial storage and processing associated with hole cutting, the current implementation in OVERFLOW stores a copy of the state vector $Q^{n}$ and Time-Spectral source term $S\left(Q^{n}\right)$ at each time level, along with an additional working copy of the grid and metrics. Thus, the total additional storage is $2 N_{x}^{3} N_{Q} N+15 N_{x}^{3}$, where $N_{x}^{3}$ and $N$ represent the number of spatial and temporal nodes, respectively, and $N_{Q}$ is the number of conserved solution variables. An additional cost of the approximate-factored matrix for the temporal derivative is also accounted for, depending upon the relative size of $N_{x}$ and $N$. Using a target problem size of $N_{x}^{3}=125 \mathrm{M}$ grid points, which is representative of a three-dimensional rotorcraft or turbomachinery simulation, and 128 8-core Intel Nehalem nodes of the NASA Advanced Supercomputing Pleiades supercomputer, Fig. 7 shows the variation in total memory with number of temporal modes for our target problem. As seen, even limiting to 128 compute nodes, with 24 GB of memory per node, there is still sufficient memory for up to 200 temporal modes, and larger problems similarly benefit from the low memory requirements.

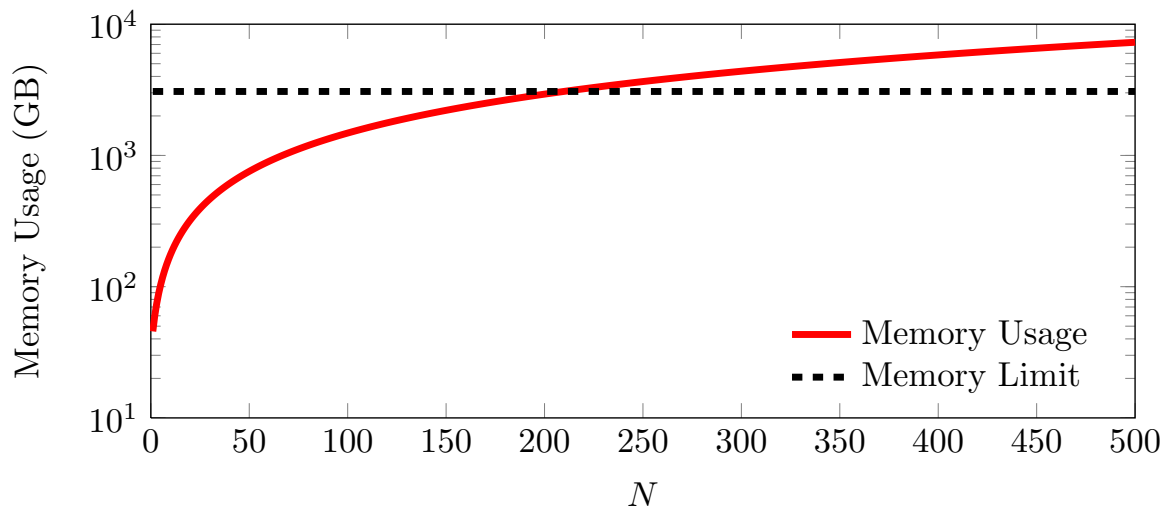

Figure 7: Memory usage estimate (GB) versus $N$ for 128 Intel Nehalem Nodes on the NASA Advanced Supercomputing (NAS) Pleiades supercomputer for a sample problem of 125 million grid points. 


\section{Numerical Results}

Two-dimensional computational studies were performed to demonstrate the ability of the hybrid TimeSpectral scheme to handle dynamic hole-cutting associated with overset relative motion. First, a largeamplitude inviscid plunging airfoil example verifies its performance in simulations involving large regions of dynamically blanked points. Next, an inviscid oscillating airfoil case corresponding to the AGARD 702 test is simulated. Finally, the turbulent case of the AGARD 702 oscillating airfoil is considered to examine how the added complexity of turbulence affects the hybrid scheme and the Time-Spectral approach in general.

Results for both rigid and relative motion were computed by the OVERFLOW Time-Spectral solver providing a direct comparison between the time-accurate approach, the conventional Time-Spectral approach, and the proposed method. These problems serve as computationally tractable demonstrations of capability and are representative of more complex three-dimensional applications.

Standard OVERFLOW inputs of second-order central differencing for the convective flux terms, first- and third-order artificial dissipation and the ARC3D Beam-Warming tridiagonal implicit spatial operator were employed. The second-order backwards difference operator (BDF2) is used to advance the time-accurate simulations. The rational polynomial order, $d$, is defined for all OVERFLOW Time-Spectral simulations as $d=\min \left(\left\lfloor\frac{N-1}{2}\right\rfloor, d_{\max }\right)$, with $d_{\max }=6$ and $N$ collocation points $($ not $N+1)$.

\section{Inviscid Plunging NACA 0012 Airfoil}

A large-amplitude inviscid plunging airfoil test case provides a meaningful demonstration of the hybrid TimeSpectral scheme as it results in a large number of dynamically blanked nodes. Plunging amplitude is chosen as half the chord length resulting in the following definition of plunging motion, $h(t)$,

$$
h(t)=\frac{c}{2} \sin (k t)
$$

with reduced frequency, $k=0.1627$ radians per non-dimensional time unit. The free stream Mach number is chosen as $M_{\infty}=0.5$ to maintain subsonic flow throughout the domain.

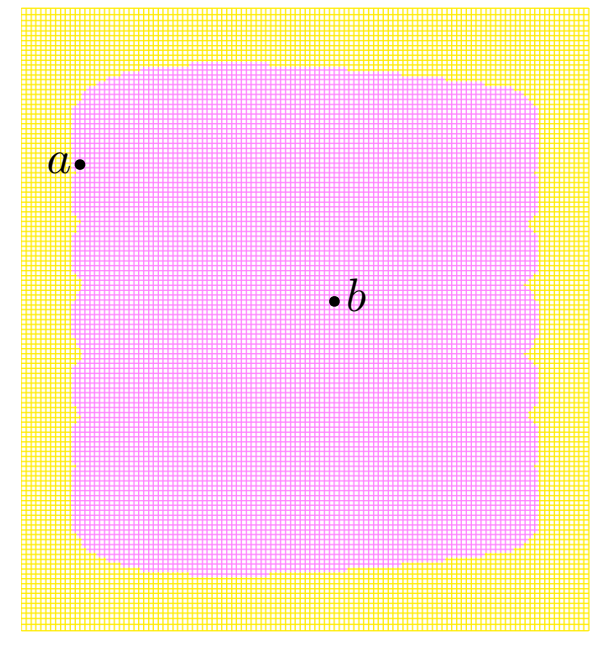

(a) Differentiation Operator Locations

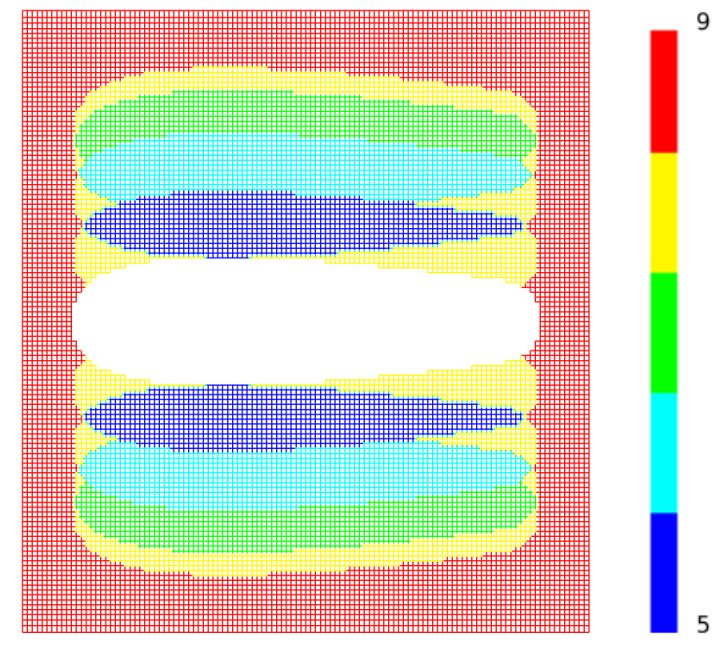

(b) Length of Temporal Interval at $t=0$

Figure 8: Plunging NACA 0012 airfoil. (a) The Fourier differentiation operator is applied in the yellow region where spatial nodes have complete time histories. The barycentric rational polynomial-based differentiation operator is applied to dynamically blanked spatial points shown in pink. Labels $a$ and $b$ locate nodes whose solutions are plotted in Figs. 9 and 10, respectively. (b) Length of time interval associated with each spatial node at the $t=0$ temporal collocation point for the $N=9$ case. The white region contains nodes blanked by the airfoil grid at the current time level.

Figure 8a shows the portion of the domain that undergoes dynamic blanking for the plunging airfoil for the case of $N=9$ time samples. The airfoil sweeps through the pink-colored region resulting in blanked spatial nodes for some subset of the period of motion. The temporal collocation points of these spatial nodes 
are therefore partitioned into intervals spanning fewer than $N=9$ time samples and differentiated with the rational polynomial operator of the appropriate rank. Nodes in the yellow-colored region remain unblanked for the duration of the period and are therefore differentiated by the Fourier operator. The length of the time interval associated with each dynamically blanked point varies and is plotted in Fig. 8b for the temporal collocation point at $t=0$. The white region shows the nodes blanked by the airfoil grid in its neutral position at $t=0$. Nodes colored red have complete time histories and correspond to the yellow-colored nodes in Fig. 8a. The solution at these nodes can be expanded in a Fourier series and are differentiated by the Fourier operator. Nodes colored yellow, green and blue are blanked for a portion of the period and only have defined solutions at a subset of the time samples. For example, nodes colored green are included in temporal intervals spanning 7 temporal collocation points including the $t=0$ time sample. The distribution of interval length changes as the number of modes is increased but this example is representative of the general case.

Figures 9 and 10 track the time history of the conserved solution variables at nodes $a$ and $b$, respectively, highlighted in Figs. 8a. Node $a$ is located near the boundary between the blanked and unblanked regions and is therefore strongly influenced by the Fourier basis. Node $a$ is blanked briefly as the airfoil sweeps upwards and again as it moves downwards resulting in two contiguous time intervals in the time-accurate case. Figure 9 plots the time-accurate and Time-Spectral solutions at node $a$. In the case of $N=5$ TimeSpectral modes, node $a$ maintains a complete time history and is therefore differentiated by the Fourier-based operator. All but one temporal collocation point is active in the $N=9$ case resulting in a single partition that is differentiated by the rational polynomial-based operator spanning eight nodes. Two independent temporal partitions are generated for the $N=17$ case resulting in one interval spanning just two temporal collocation points and another spanning an additional twelve collocation points. Excellent agreement for all the modal cases is observed with only small deviations from the time-accurate solution. Node $b$ located in the center of the blanked region is farther from Fourier-based nodes. The continuous solution at node $b$ is partitioned into two intervals of similar length. Only the first temporal collocation point is blanked for the $N=5$ and $N=9$ Time-Spectral cases whereas the time history of the $N=17$ case is partitioned into two intervals, each spanning seven time samples. The time-accurate and Time-Spectral solutions at node $b$ are plotted in Fig. 10. Larger discrepancies between the time-accurate and Time-Spectral solution of streamwise momentum, $\rho u$, are manifested for the $N=5$ and $N=9$ cases (Fig. 10b) but the solution does converge with increased temporal resolution evidenced by the $N=17$ case. 


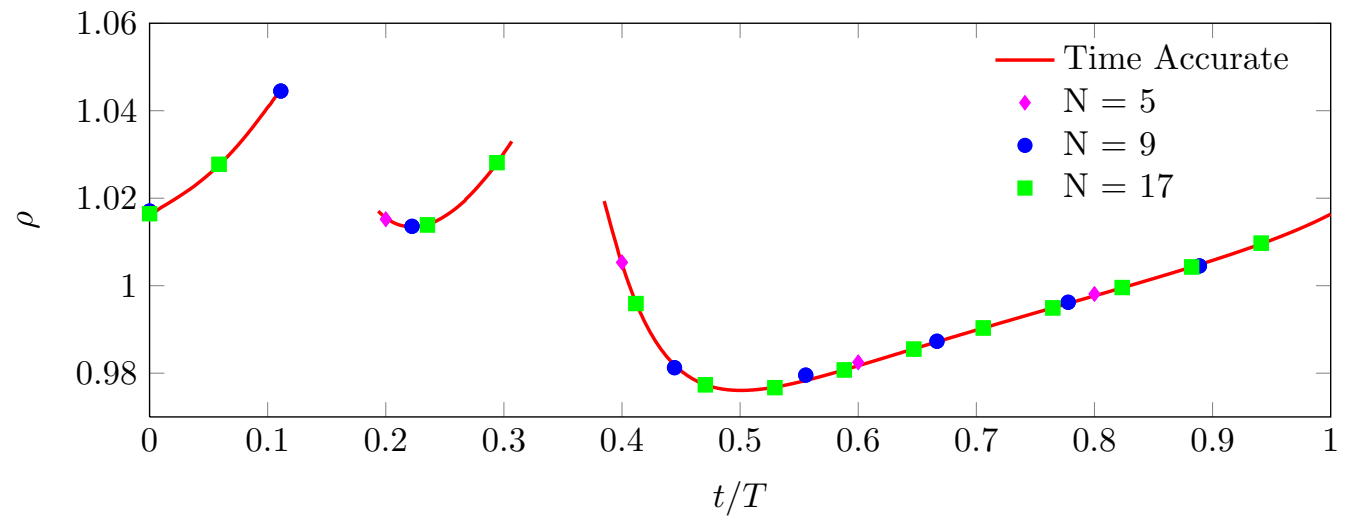

(a) Density, $\rho(t)$

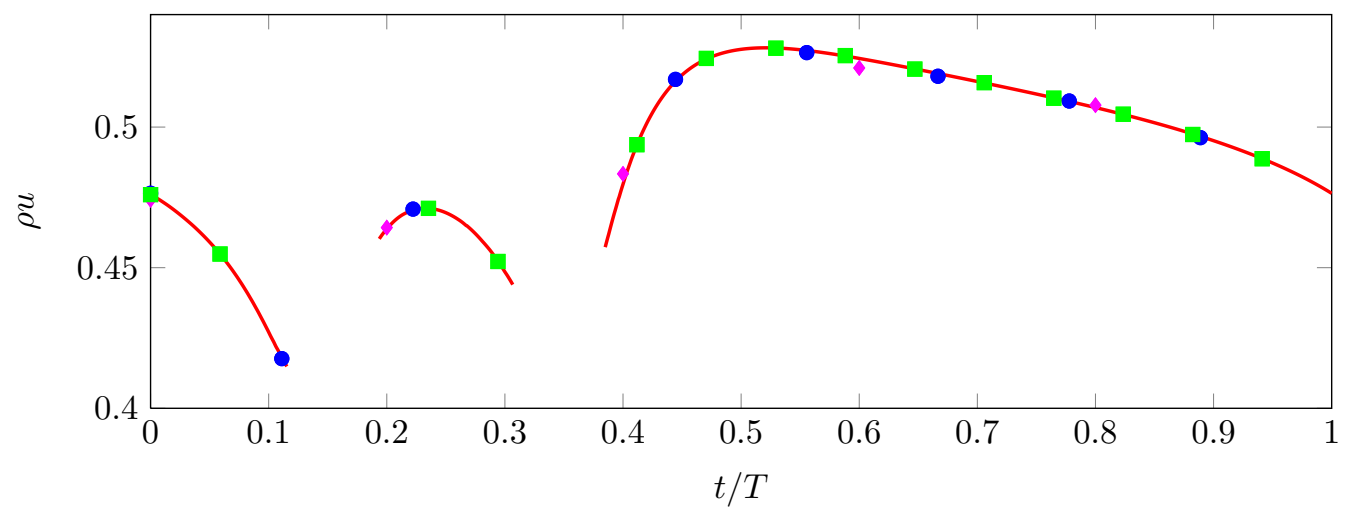

(b) $x$-Momentum, $\rho u(t)$

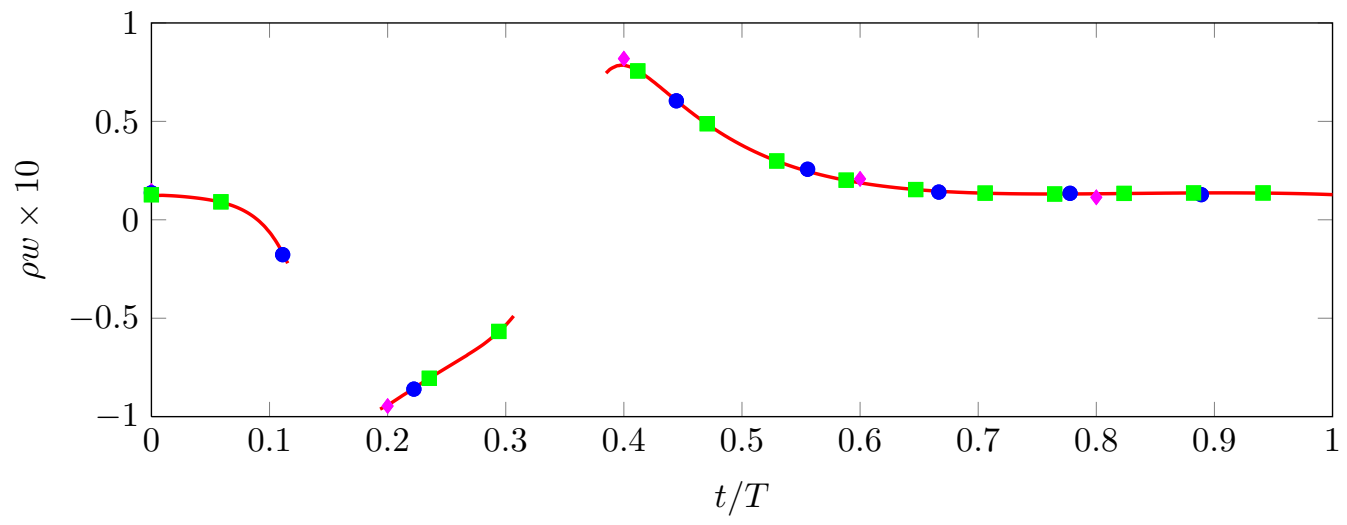

(c) $z$-Momentum, $\rho w(t)$

Figure 9: Time-accurate and Time-Spectral solutions of $\rho, \rho u$ and $\rho w$ for the plunging NACA 0012 airfoil at node $a$ in Fig. 8a. Values from the Time-Spectral solutions using $N \in\{5,9,17\}$ time samples are plotted at their respective collocation points against the time-accurate solutions. 


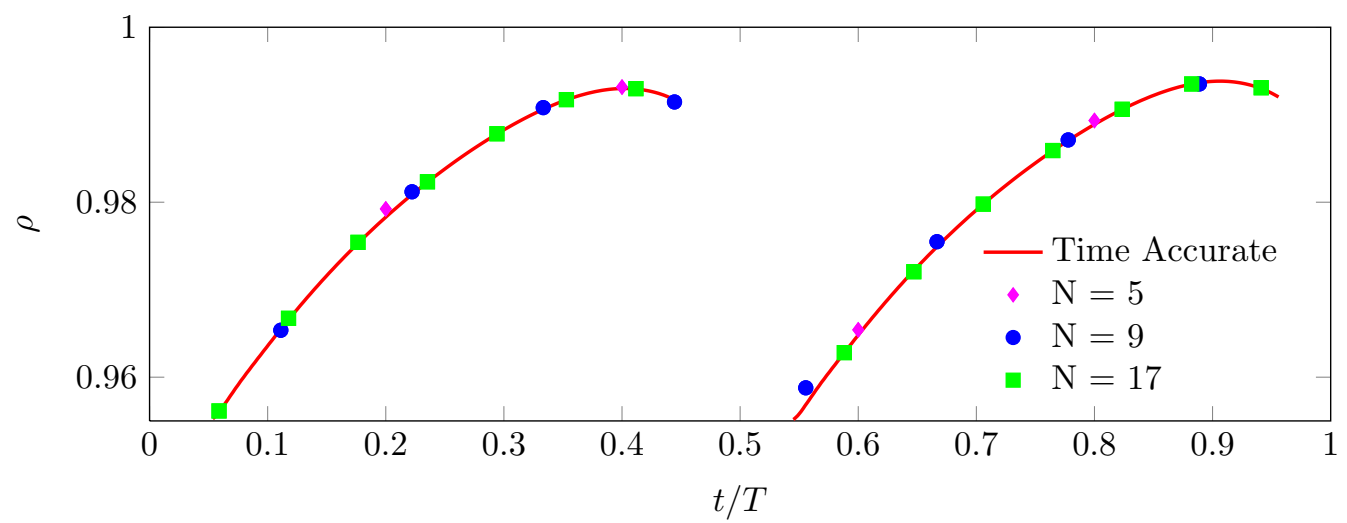

(a) Density, $\rho(t)$

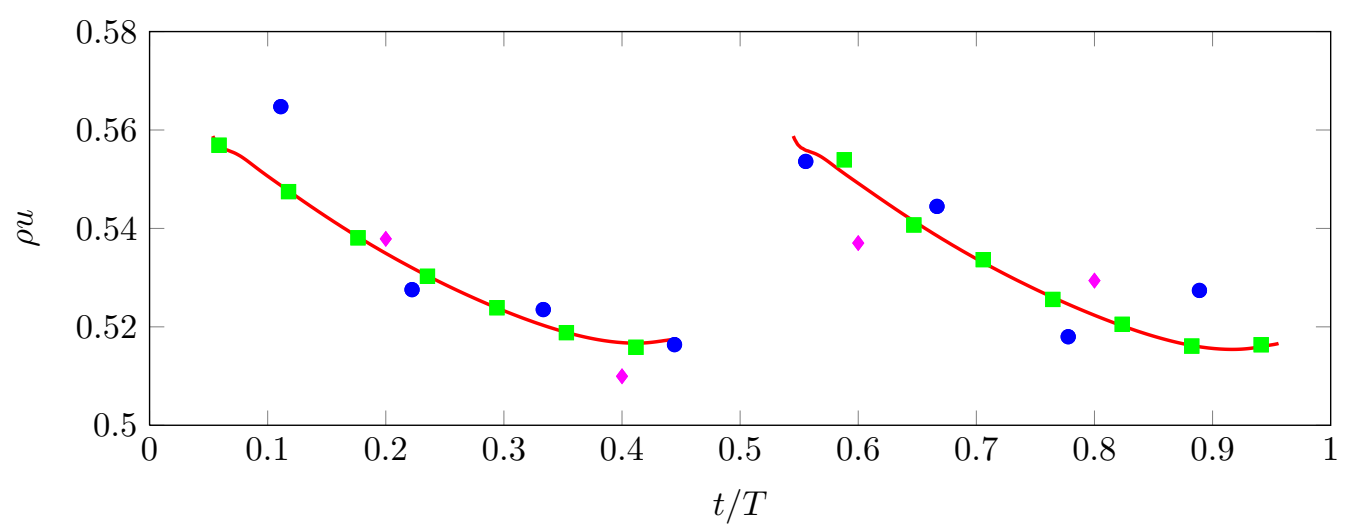

(b) $x$-Momentum, $\rho u(t)$

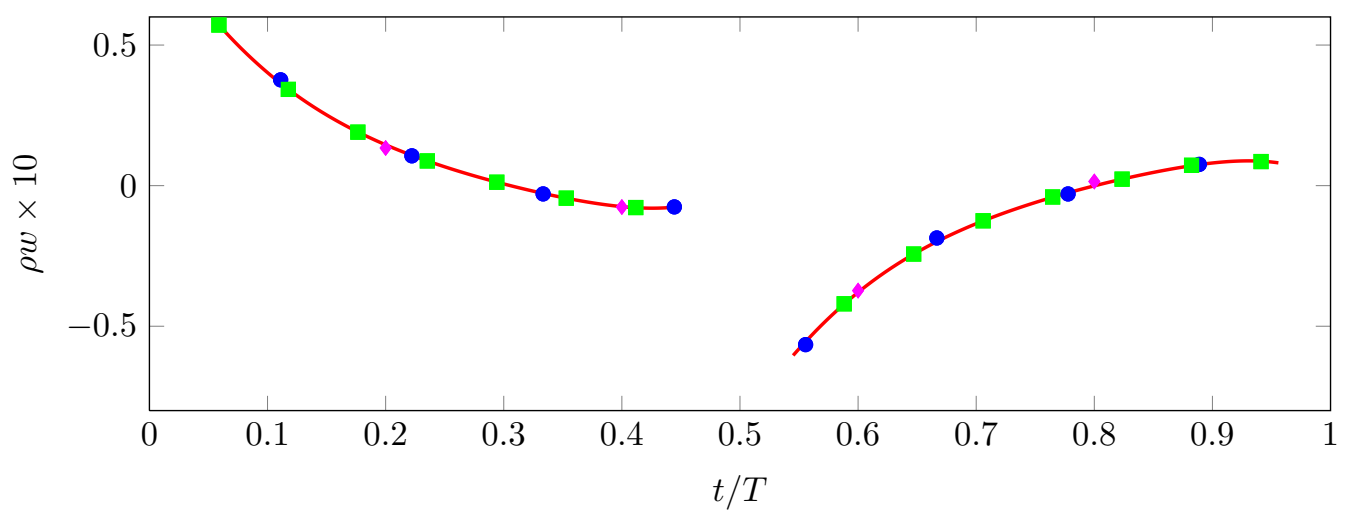

(c) $z$-Momentum, $\rho w(t)$

Figure 10: Time-accurate and Time-Spectral solutions of $\rho, \rho u$ and $\rho w$ for the plunging NACA 0012 airfoil at node $b$ in Fig. 8a. Values from the Time-Spectral solutions using $N \in\{5,9,17\}$ time samples are plotted at their respective collocation points against the time-accurate solutions. 


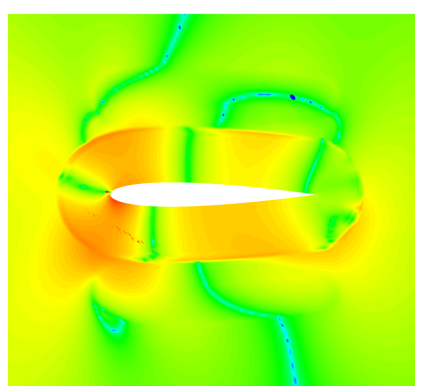

(a) $N=5$

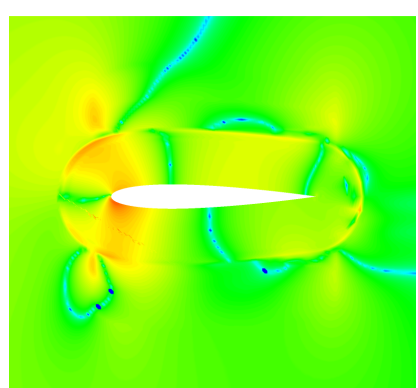

(b) $N=9$

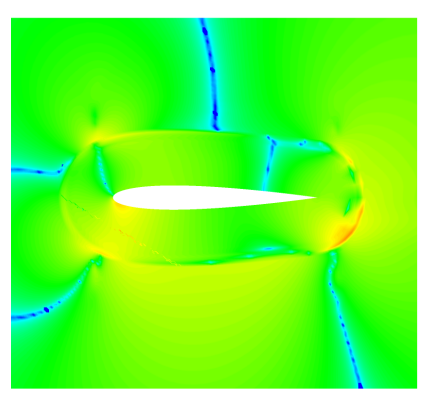

(c) $N=17$

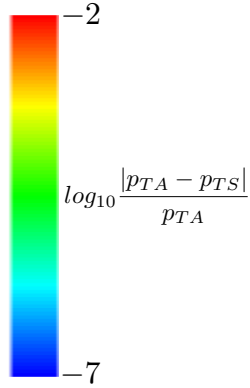

Legend

Figure 11: Subsonic plunging NACA 0012 airfoil. Normalized error in pressure, $\operatorname{err}_{p}=\frac{\left|p_{T A}-p_{T S}\right|}{p_{T A}}$, of the Time-Spectral versus time-accurate solutions at $t=0$ for $N \in\{5,9,17\}$. Error is plotted on a $\log$ scale ranging from -2 to -7 suggesting that even the $N=5$ case has less than $1 \%$ error in pressure over then entire flow-field at the $t=0$ instance.

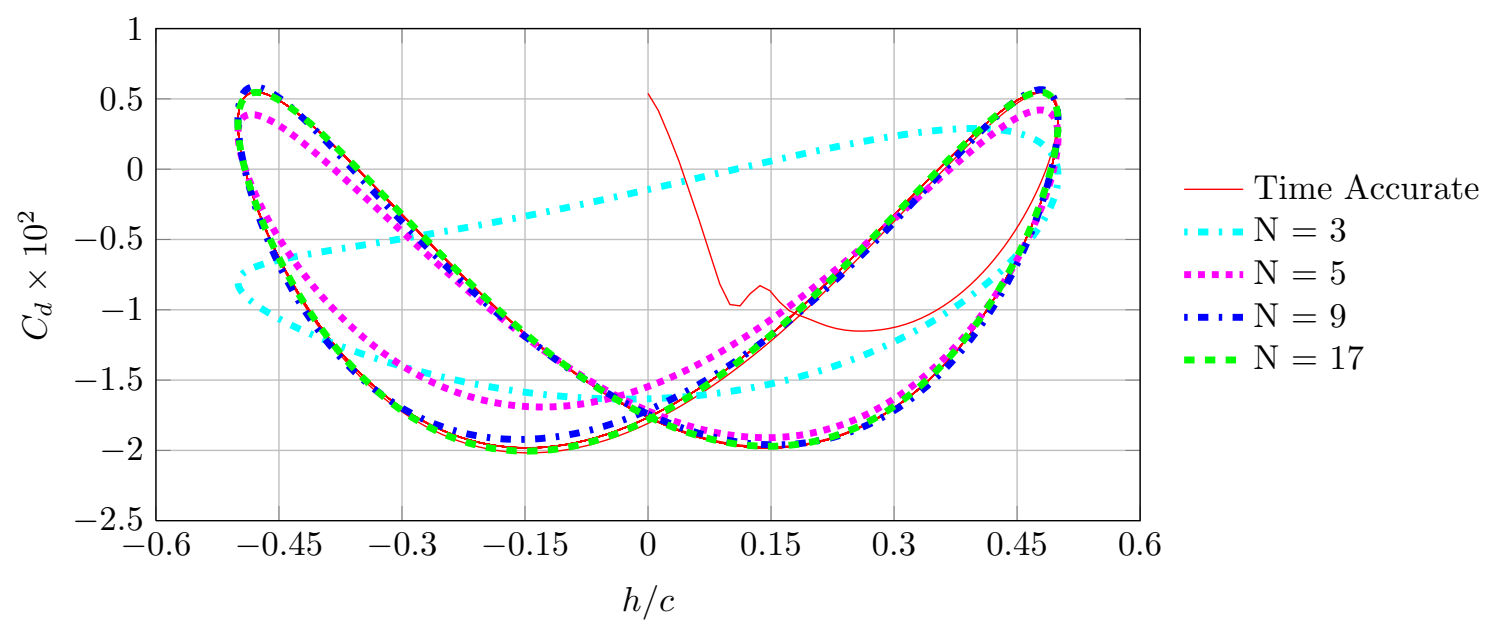

Figure 12: Subsonic plunging NACA 0012 airfoil. Convergence of relative-motion Time-Spectral projection of drag coefficients towards time-accurate solution for $N \in\{3,5,9,17\}$. The Time-Spectral drag signals for the $N=9$ and $N=17$ cases are observed to nearly match the time-accurate solution demonstrating temporal convergence.

To examine solution quality on a larger scale the global error in pressure is computed taking the relativemotion time-accurate pressure, $p_{T A}$, as exact. Normalized error in pressure, $\operatorname{err}_{p}$, is visualized in Fig. 11 for $N \in\{5,9,17\}$ at time $t=0$ which is the only common temporal collocation point among the three examples. The maximum normalized error in pressure occurs in the $N=5$ flow field but is limited to roughly one part in 1000. The error is reduced monotonically by increasing the number of modes. This is evident in the drag coefficient in Fig. 12. The $N=3$ and $N=5$ Time-Spectral cases demonstrate differences from the time-accurate result, but the $N=9$ case shows improvement and the $N=17$ case matches the time-accurate solution nearly identically.

\section{Transonic AGARD 702 Pitching Airfoil}

The Time-Spectral formulation is next compared to time-accurate simulations and experimental data corresponding to the AGARD 702 3E3 oscillatory pitch test case CT5 [34]. Both inviscid and viscous turbulent simulations are performed on this transonic, $M_{\infty}=0.755$, case. A pitching airfoil allows for simulations with both rigid- and relative-body motion so that the temporal discretization can be isolated and compared directly. The airfoil pitches about its quarter chord with incidence, $\alpha(t)$, defined as

$$
\alpha(t)=0.016^{\circ}+2.51^{\circ} \sin (k t)
$$


with reduced frequency $k=0.1627$ radians per non-dimensional time unit and free stream Mach number $M_{\infty}=0.755$. A hierarchy of Time-Spectral simulations are computed using an increasing number of modes $(N \in\{3,5,9,17,33\})$ to investigate the performance of the scheme with increased temporal resolution and ensure that spurious higher-frequency modes are not generated and propagated into the solution. The nearbody grid and its overlap region are chosen such that the transient shock spans the grid interface. Figure 13 shows the supersonic region and the overset-grid system at two of the temporal collocation points for the $N=5$ case.

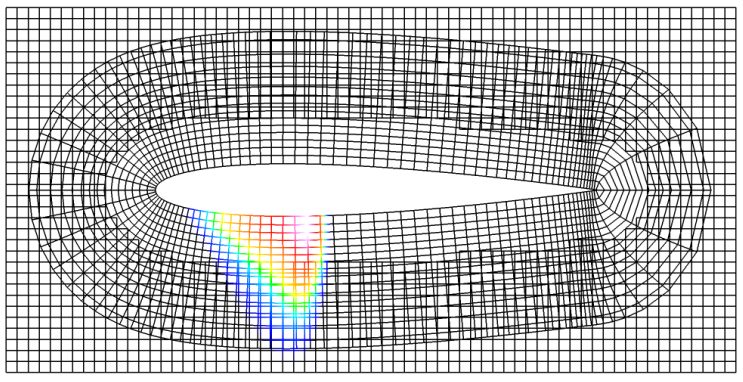

(a) $\frac{t N}{T}=0$

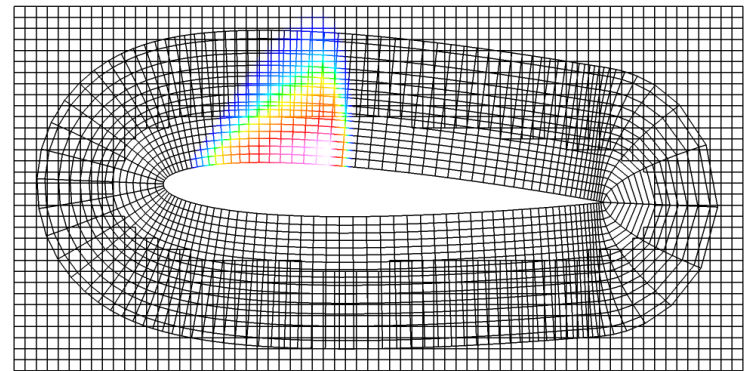

(b) $\frac{t N}{T}=1$

Figure 13: AGARD 702 inviscid pitching airfoil at $M_{\infty}=0.755$. The supersonic region is plotted on the grid-system in the vicinity of the airfoil for two time samples of the $N=5$ case demonstrating the shock spanning the near-body to off-body grid interface. Subsonic regions shown in black. Every second grid point has been removed in the figure for clarity.

\section{Inviscid Case}

A $241 \times 30$ O-mesh near-body grid is embedded in a $341 \times 261$ rectilinear background grid that stretches approximately 100 chord lengths to the far field in both directions. In the case of rigid-body motion both the near-body and background grids rotate together. This provides constant hole-cutting permitting the use of the conventional Time-Spectral approach using the Fourier-based differentiation operator. In the relative-motion case, the near-body grid rotates independently of the background grid introducing dynamic hole-cutting. The unsteady residual was converged to machine precision at each physical time step in the time-accurate case. The complete space-time residual for the Time-Spectral cases also converges to machine precision (Fig. 14). The convergence rate is independent of the number of Time-Spectral modes in this case.

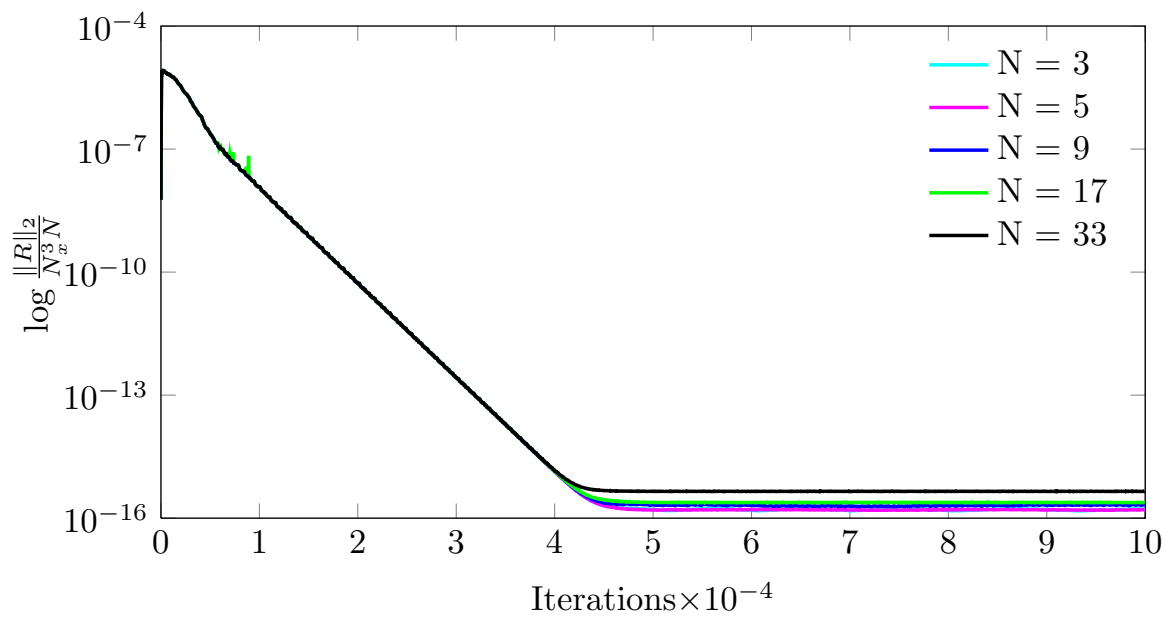

Figure 14: AGARD 702 inviscid pitching airfoil. Iterative convergence of space-time residual for relativebody motion for $N \in\{3,5,9,17,33\}$. The sum of both the near- and off-body grid residuals normalized by the total number of points in the domain is plotted versus iteration number. 
Pitching moment and drag coefficient polars are plotted in Figs. 15 and 16, respectively. The rigid- and relative-motion Time-Spectral solutions and the time-accurate case, all converge to the identical solution. Reasonable agreement with the experimental data is achieved but most importantly, significant temporal resolution convergence of the hybrid Time-Spectral solutions is achieved as the number of sample points is increased from 3 to 33 allowing the solution to resolve increasingly higher-frequency content inherent in the unsteady solution and its output functionals. Note that 33 modes are required to resolve the kink in the pitching moment and drag signals at $\alpha \pm 2^{\circ}$. Root-mean-square error between the unsteady force and pitching moment coefficients of the rigid- and relative-motion case isolates the quantitative effect of the hybrid differentiation operator on the Time-Spectral solution and confirms the convergence (Table 1).

\begin{tabular}{cccc}
$\mathrm{N}$ & $C_{l}$ & $C_{d}$ & $C_{m}$ \\
\hline 3 & $1.96 \mathrm{e}-03$ & $3.64 \mathrm{e}-04$ & $6.24 \mathrm{e}-04$ \\
5 & $3.50 \mathrm{e}-04$ & $3.83 \mathrm{e}-05$ & $2.01 \mathrm{e}-04$ \\
9 & $7.83 \mathrm{e}-04$ & $1.24 \mathrm{e}-05$ & $4.22 \mathrm{e}-05$ \\
17 & $6.98 \mathrm{e}-05$ & $1.03 \mathrm{e}-05$ & $1.68 \mathrm{e}-05$ \\
33 & $4.50 \mathrm{e}-05$ & $4.08 \mathrm{e}-06$ & $1.39 \mathrm{e}-05$ \\
\hline
\end{tabular}

Table 1: Root-mean-square error in force and pitching moment coefficients between projections of rigid- and relative-motion Time-Spectral solutions.

The frequency components, amplitude and phase, of the time-accurate pitching moment coefficient are plotted in Fig. 17 against those computed by the hybrid Time-Spectral scheme. Figure 17a reveals that the $N=3$ case fails to resolve even the mean amplitude and the $N=5$ case poorly resolves the either the amplitude or phase corresponding to the second harmonic. Increasing the number of solution modes permits resolution of higher frequency content, and improves resolution of the lower frequencies. Importantly, no artificial high-frequency amplitude or phase content corrupts the solution as a result of the hybrid TimeSpectral scheme so that increasing the number of solution modes results in solutions that mirror the frequency content of the time-accurate simulation. 


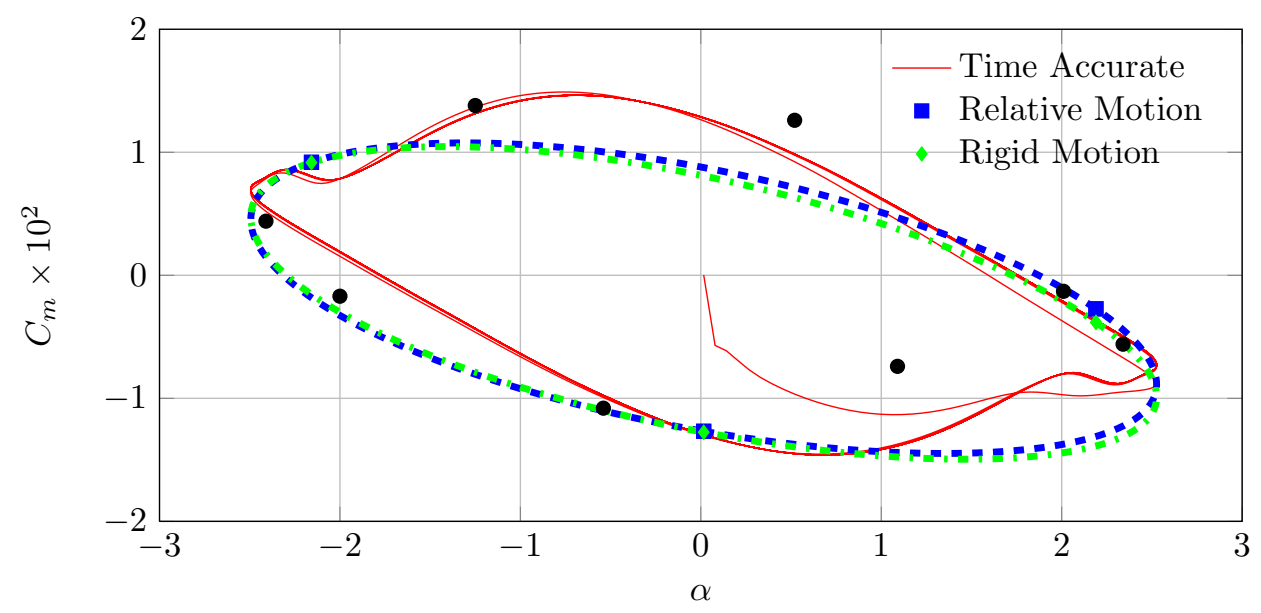

(a) $N=3$

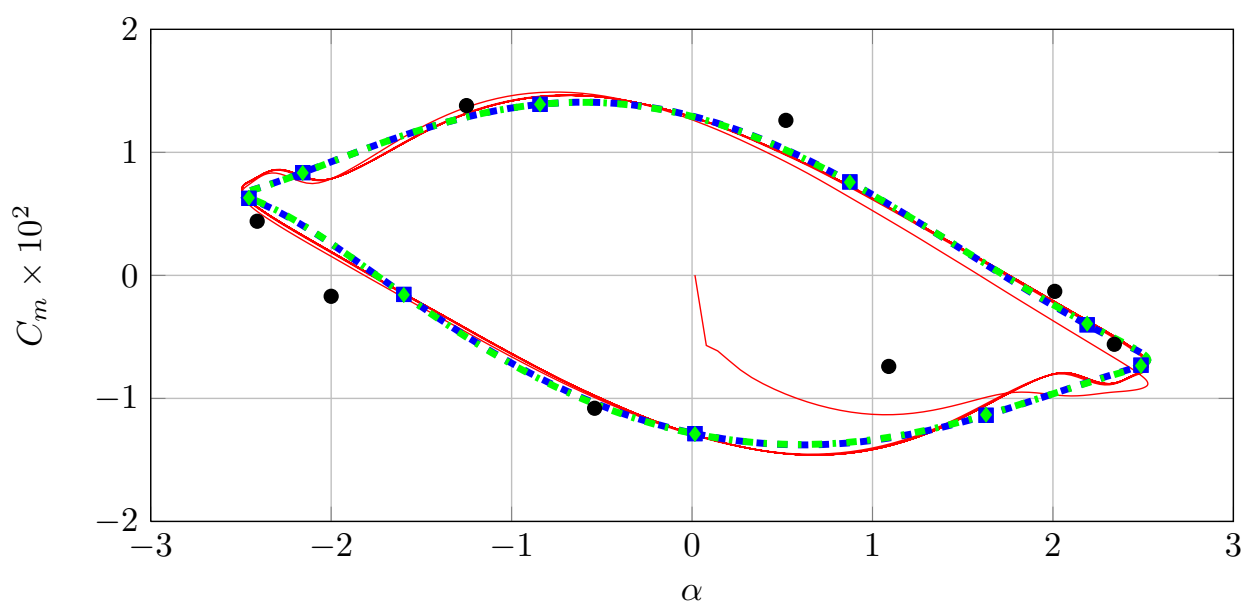

(b) $N=9$

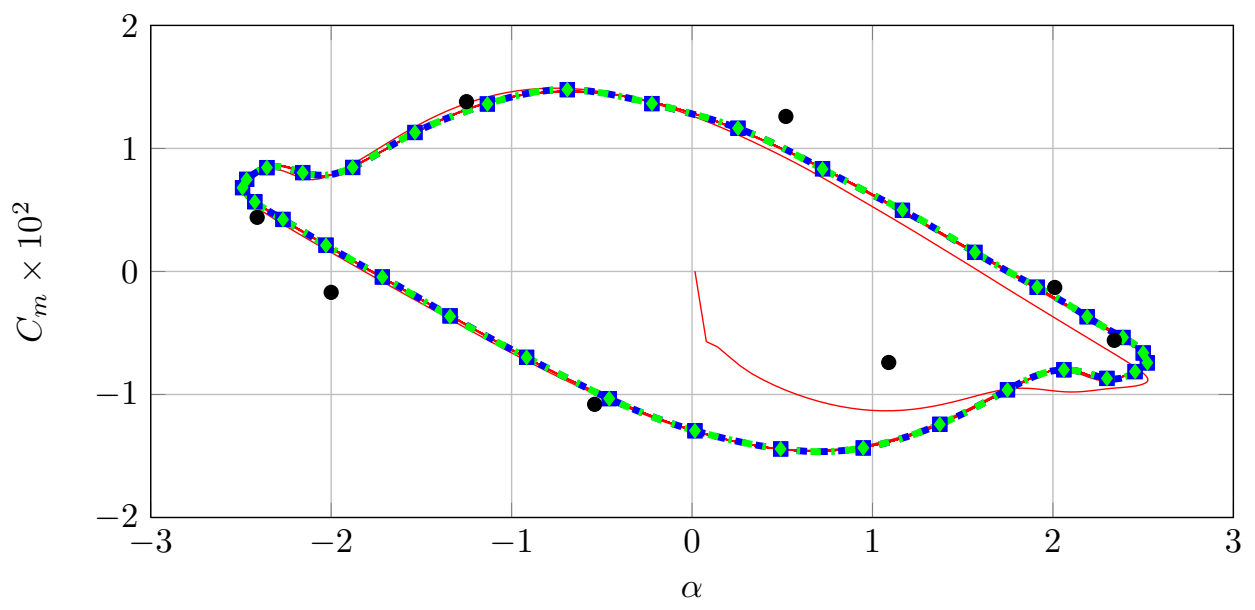

(c) $N=33$

Figure 15: AGARD 702 inviscid pitching airfoil. Time Spectral versus Time Accurate pitching moment coefficients for $N \in\{3,9,33\}$. Ten periods of the time-accurate solution are plotted in red from steady-state startup. Blue squares locate the pitching moment coefficient values at the Time-Spectral collocation points for relative-body motion. Relative-body pitching moment coefficients computed from an interpolation of the Time-Spectral solution to 201 points shown with the blue-hashed line. Green diamonds locate the pitching moment coefficient values at the Time-Spectral collocation points for rigid-body motion. Rigid-body pitching moment coefficients computed from an interpolation of the Time-Spectral solution to 201 points shown with the green-hashed line. Experimental data from the AGARD 702 Report is plotted with black dots. 


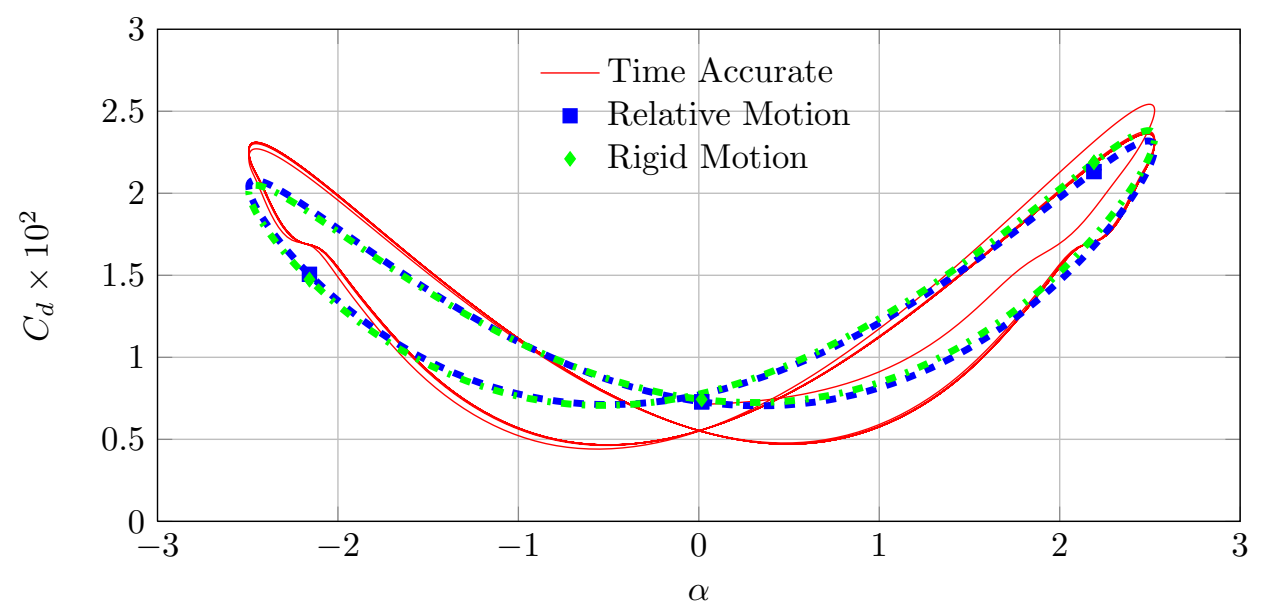

(a) $N=3$

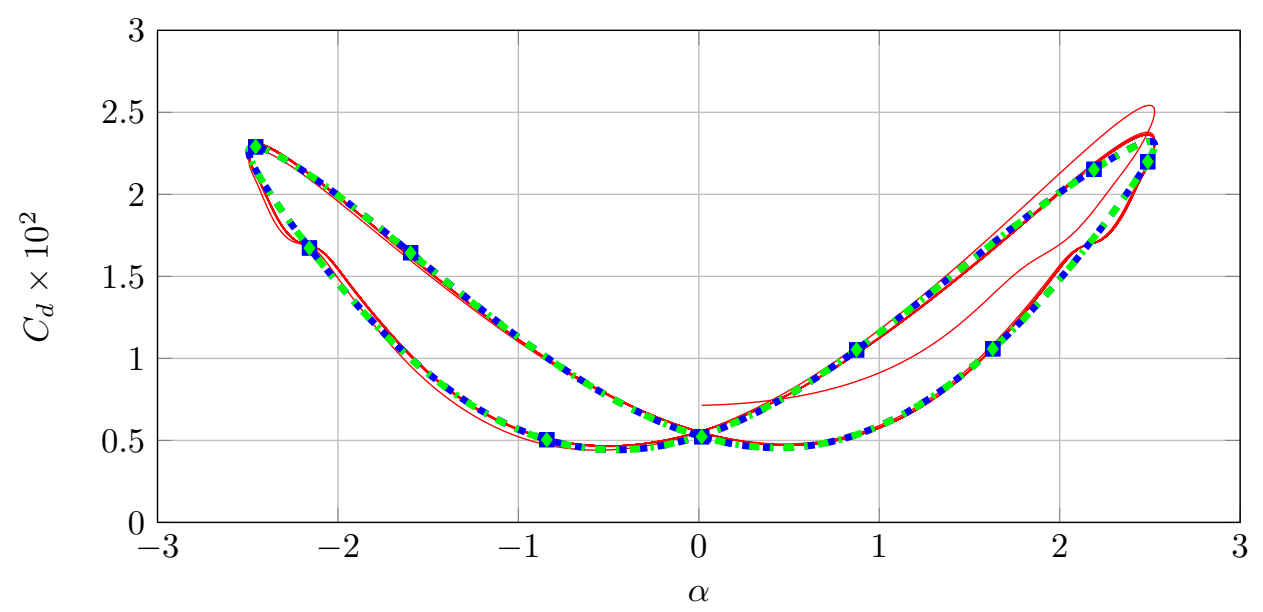

(b) $N=9$

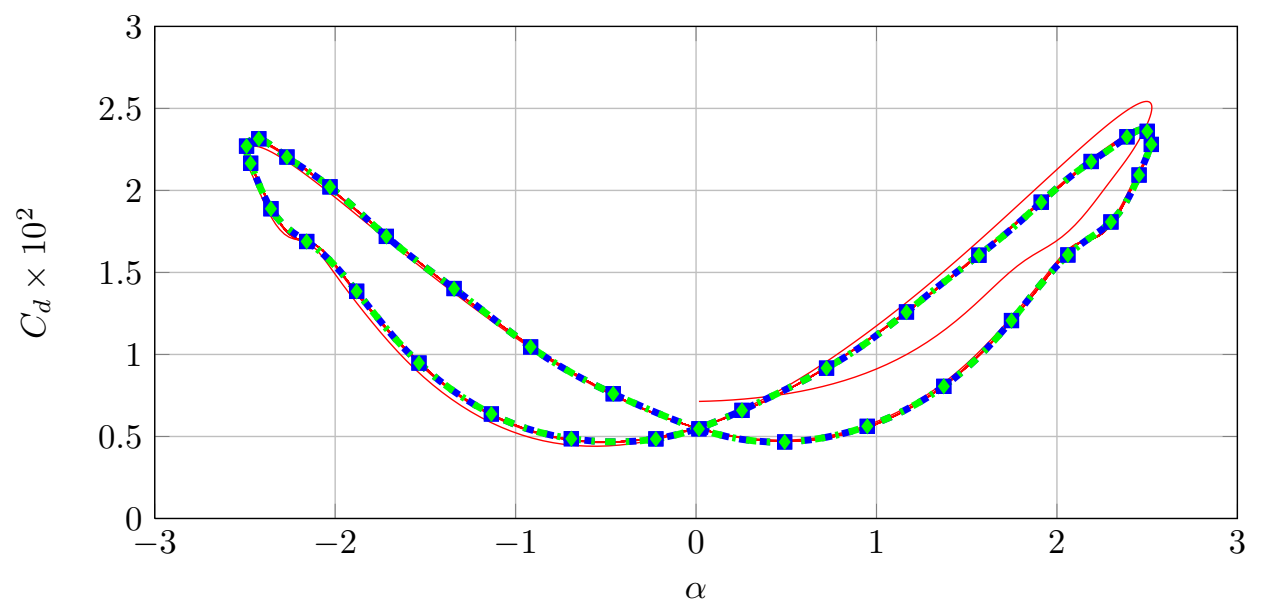

(c) $N=33$

Figure 16: AGARD 702 inviscid pitching airfoil. Time Spectral versus Time Accurate drag coefficients for $N \in\{3,9,33\}$. Ten periods of the time-accurate solution are plotted in red from steady-state startup. Blue squares locate the drag coefficient values at the Time-Spectral collocation points for relative-body motion. Relative-body drag coefficients computed from an interpolation of the Time-Spectral solution to 201 points shown with the blue-hashed line. Green diamonds locate the drag coefficient values at the Time-Spectral collocation points for rigid-body motion. Rigid-body drag coefficients computed from an interpolation of the Time-Spectral solution to 201 points shown with the green-hashed line. Corrected experimental drag data from the AGARD 702 Report is not available. 


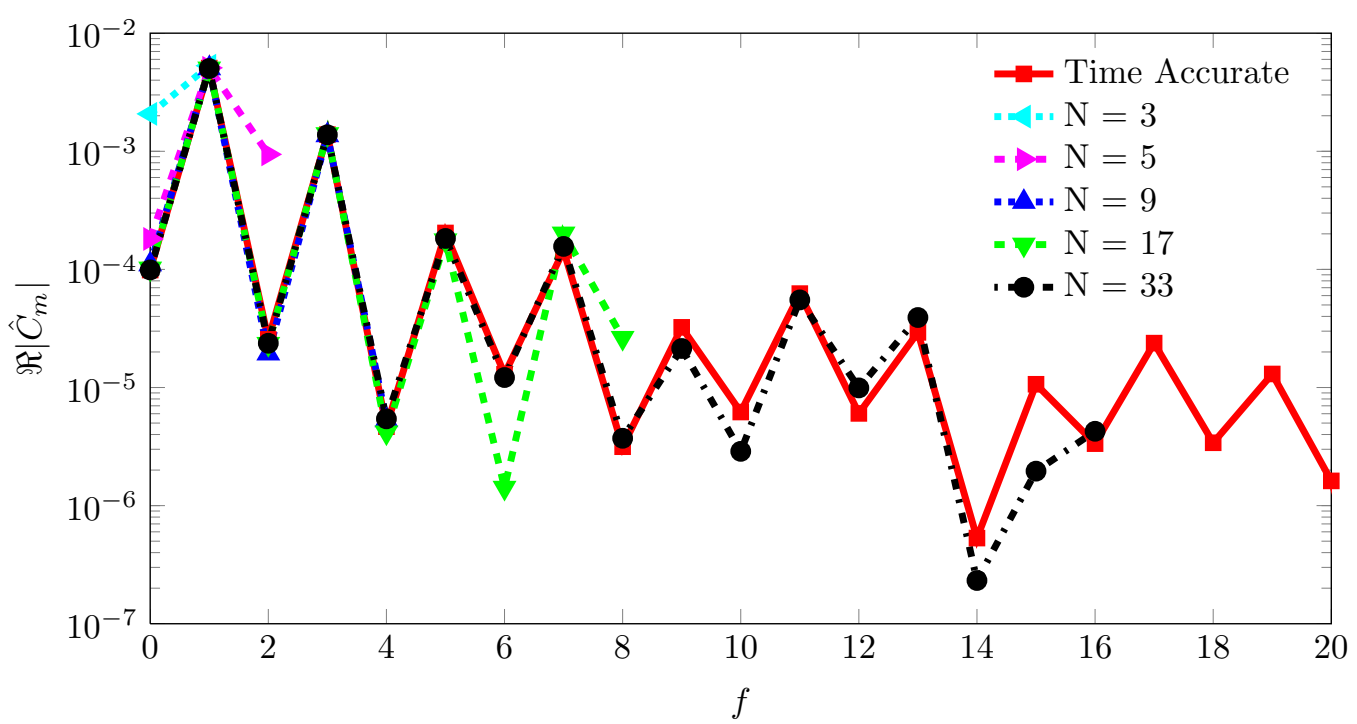

(a) Amplitude, $\Re\left|\hat{C}_{m}\right|$

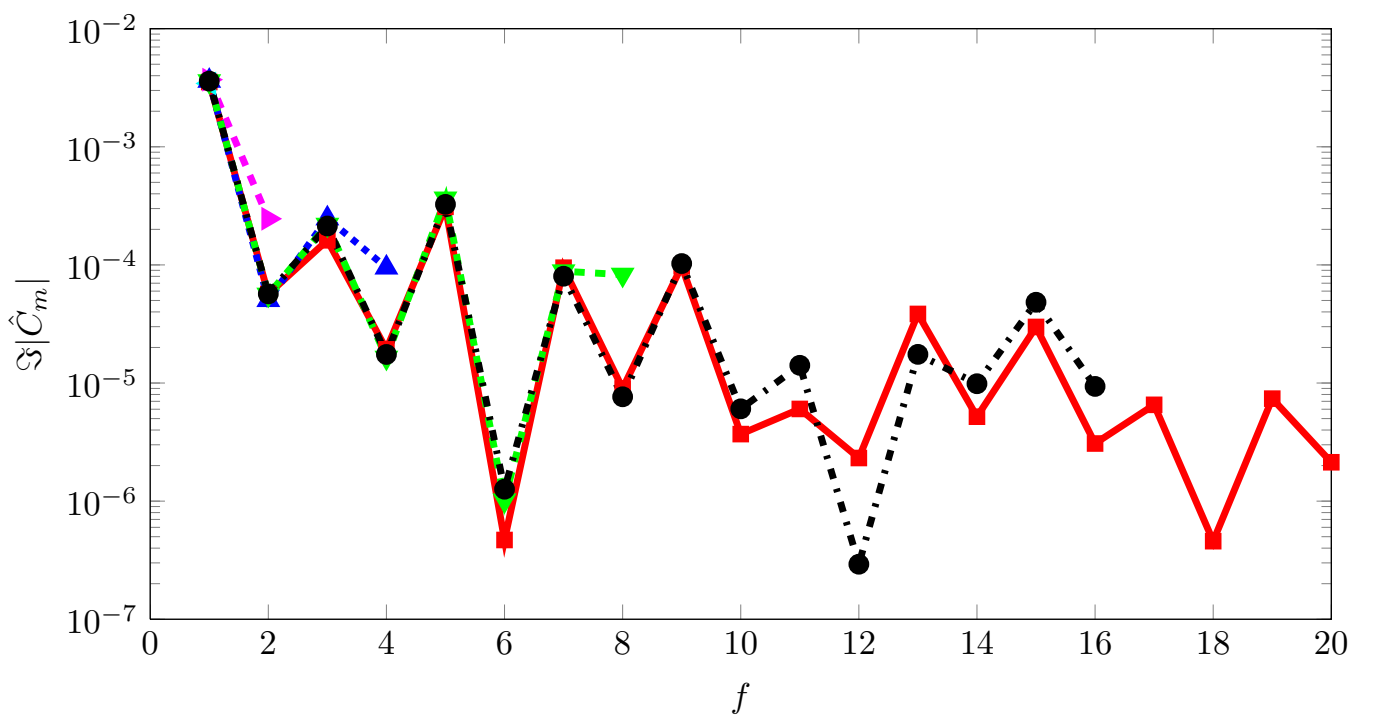

(b) Phase, $\Im\left|\hat{C}_{m}\right|$

Figure 17: AGARD 702 inviscid pitching airfoil. Spectrum of time-accurate and Time-Spectral pitching moment coefficients, $\hat{C}_{m}=\boldsymbol{\Phi}^{-1} C_{m}$, for first 20 harmonics of the relative motion case. Increasing modal resolution results in better agreement between output functional amplitude and phase without introducing spurious high-frequency content.

\section{Turbulent RANS Case}

Practical applications of the Time-Spectral method including rotorcraft and turbomachinery involve turbulent flows, and therefore the previous oscillatory airfoil is extended to use the Spalart-Allmaras one-equation turbulence model. OVERFLOW employs a loosely coupled turbulence scheme whereby the turbulent variables are updated initially and held constant for the flow equation iteration. The code structure prevents a direct Time-Spectral implicit treatment for the turbulent variable without a significant overhaul. Instead, a semi-implicit treatment retroactively applies the implicit operator to the explicit turbulent update, $\Delta \tilde{\nu}^{n+\frac{1}{2}}$.

$$
\left[I+\Delta \tau A_{x}\right]\left[I+\Delta \tau A_{y}\right]\left[I+\Delta \tau A_{z}\right] \Delta \tilde{\nu}^{n+\frac{1}{2}}=-\Delta \tau\left[R\left(\tilde{\nu}^{n}\right)+S\left(\tilde{\nu}^{n}\right)\right]
$$


$\tilde{\nu}^{n+\frac{1}{2}}$ is then held fixed while iterating the flow solution, $\vec{Q}$.

$$
\vec{Q}^{n+1}=\vec{Q}^{n}+\Delta \vec{Q}\left(\tilde{\nu}^{n+\frac{1}{2}}\right)
$$

The implicit update $\Delta \tilde{\nu}^{n+1}$ is computed and retroactively applied to $\tilde{\nu}^{n}$ to advance the solution prior to the subsequent time step.

$$
[I+\Delta \tau \mathcal{D}] \Delta \tilde{\nu}^{n+1}=\Delta \tilde{\nu}^{n+\frac{1}{2}}
$$

Figure 18 shows contours of the (undamped) turbulent eddy viscosity, $\tilde{\nu}$, in the wake region for the five temporal collocation points of the pitching airfoil. This illustrates the potentially large changes in $\tilde{\nu}$ as a function of time in the wake region.

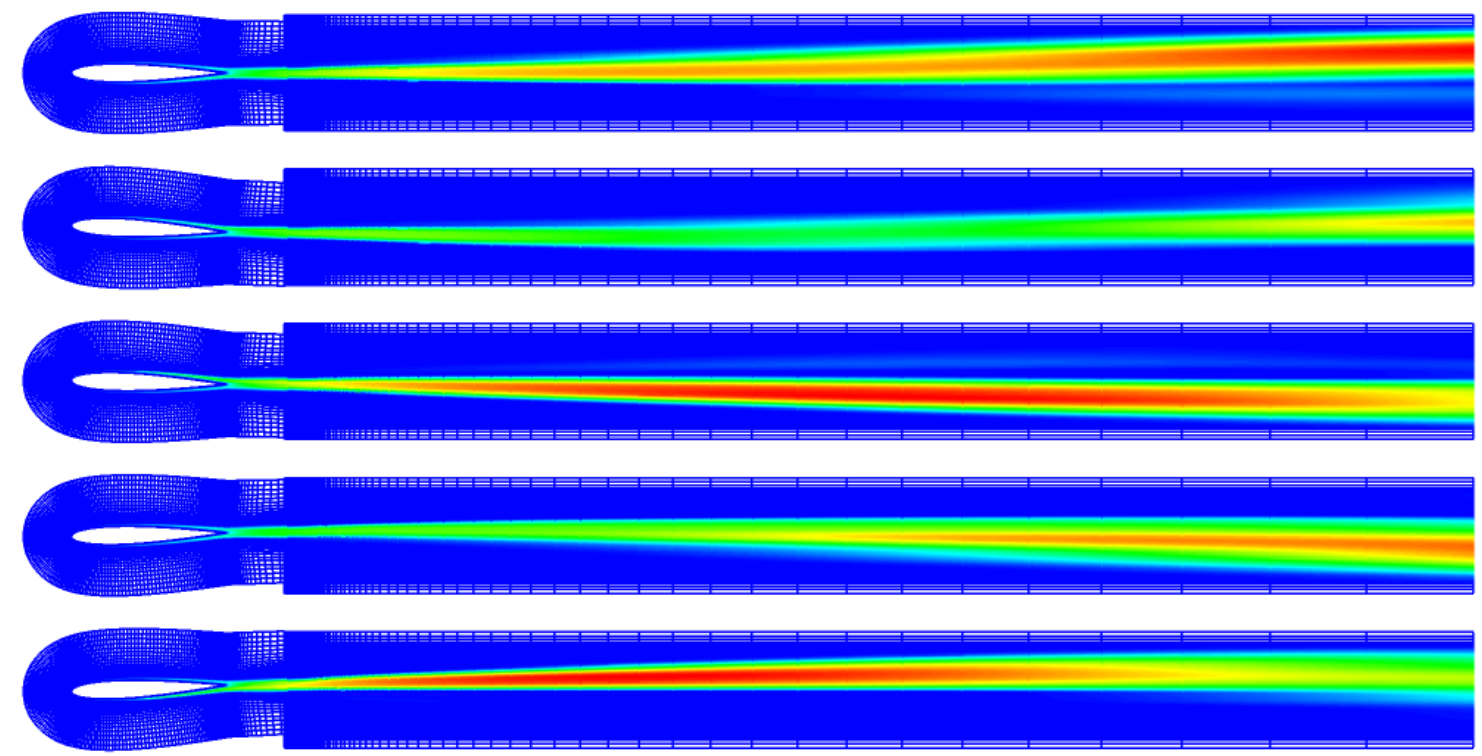

Figure 18: Visualization of undamped turbulent eddy viscosity on the near-body grid and in the wake region of the AGARD 702 pitching airfoil at the temporal collocation points for a representative case of $N=5$. $\tilde{\nu}$ can vary several orders of magnitude in a short time-frame and must be limited in certain circumstances to avoid spurious undershoots resulting from Gibbs' phenomenon. The figure shows every second grid point removed in the normal direction for clarity.

Figure 19 plots the time history of turbulent eddy viscosity and its temporal derivative at a point downstream of the airfoil through which the turbulent wake passes during a portion of the oscillation. Note in Fig. 19a, the turbulent eddy viscosity transitions from a constant value of order zero to a value of order 1000 over a small fraction of the period. While the function varies smoothly in the context of the small physical time steps associated with time-accurate iterations, it resembles a discontinuous step function in the frame of the much coarser resolution of Time-Spectral sample points. This leads to spurious oscillations in the Fourier expansion (Gibbs' phenomenon). Oscillations in the gradient are manifested at the discrete sample points which can be observed in Fig. 19b resulting in large overshoots. This issue is compounded by the fact that undamped eddy viscosity remains close to zero outside the wake, so that modest overshoots in the derivative violate the positivity constraint on $\tilde{\nu}$. The current example uses eddy viscosity in the Spalart-Allmaras model, however, similar issues are encountered for turbulent kinetic energy or dissipation in other models. The same phenomenon could also occur in the fluid equations for high-speed cases. Note that this issue applies to Time-Spectral treatment of turbulence in general as it occurs downstream from the body on nodes with complete time histories.

Applying a limiter to the temporal differentiation operator maintains positivity on $\tilde{\nu}$ without sacrificing accuracy. The current limiter sets the Time-Spectral source term to zero if it would drive $\tilde{\nu}$ negative. Figure 19a demonstrates good agreement between the Time-Spectral and time-accurate solutions for $\tilde{\nu}$ at the collocation points. Slight ringing does occur in the constant region but $\tilde{\nu}$ remains positive. 


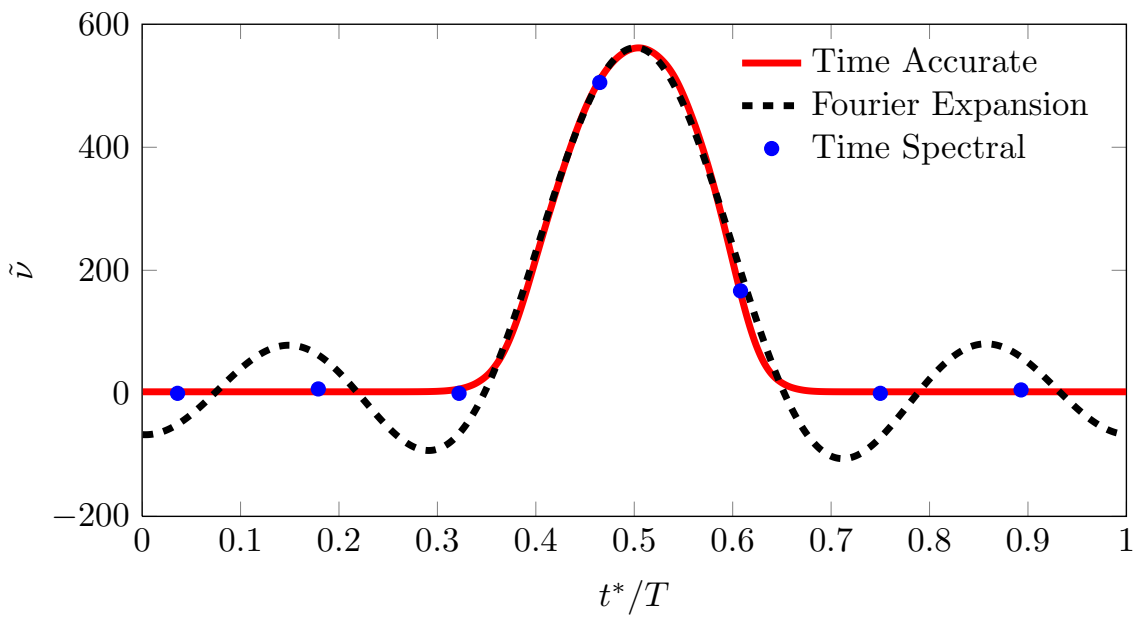

(a) Turbulent variable, $\tilde{\nu}(t)$

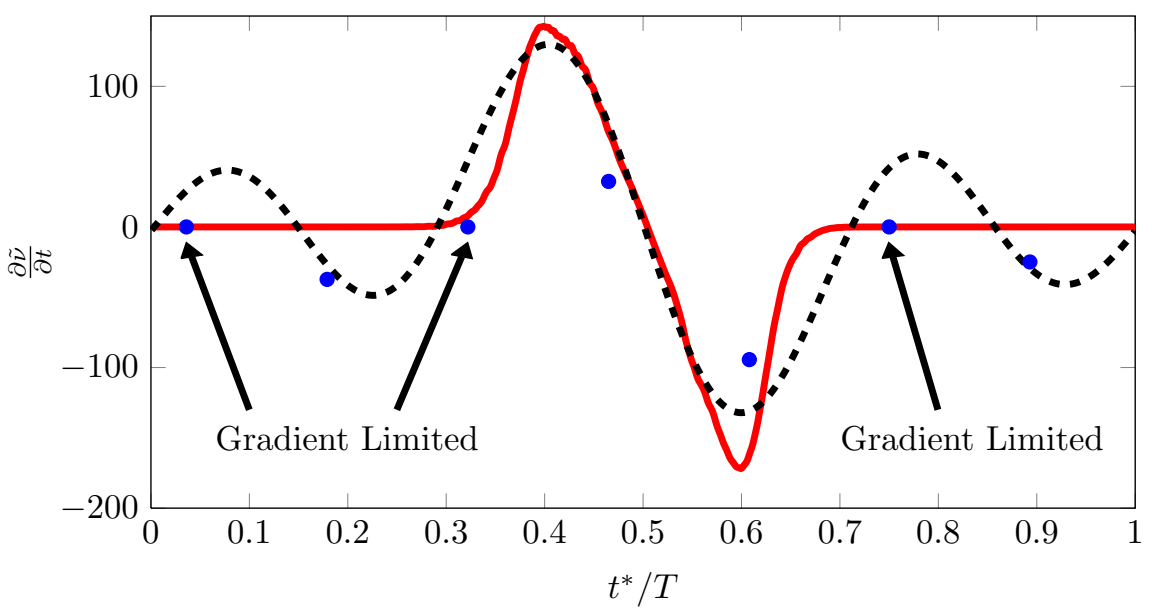

(b) Time derivative, $\frac{\partial \tilde{\nu}}{\partial t}(t)$

Figure 19: AGARD 702 turbulent pitching airfoil. (a) Turbulent solution variable, $\tilde{\nu}(t)$, and (b) its derivative, $\frac{\partial \tilde{\nu}}{\partial t}$, are plotted for a point in the wake over the period of oscillation. BDF2 and Fourier operators differentiated the continuous and discrete data, respectively. Note, Gibbs' phenomenon in the Fourier representation of the signal are present in the derivative where large oscillations lead to an inaccurate representation of $\frac{\partial \tilde{\nu}}{\partial t}$ $\left(t^{*}=t-T / 4\right)$.

Only the relative-motion Time-Spectral scheme is applied to the viscous case as the hybrid scheme already demonstrated its ability to match the results computed with the conventional Time-Spectral scheme in inviscid rigid-body case. The drag and pitching moment coefficients computed from the Time-Spectral simulations converge to those computed in unsteady mode with increasing numbers of temporal collocation points (Figs. 20 and 21). Note that $N=33$ modes are required to resolve the kink in the drag and pitching moment coefficient signals at $\alpha \approx \pm 2^{\circ}$ (Figs. 20c and 21c), as with the inviscid case. Less agreement is observed with the experimental data in the pitching moment coefficient polars (Fig. 20) when compared to the inviscid case from the previous section but this discrepancy also occurs in the time-accurate case indicating suggesting a modeling issue. While experimental data is included for reference, the crucial point remains that the Time-Spectral results matched those computed with the time-accurate solver. 


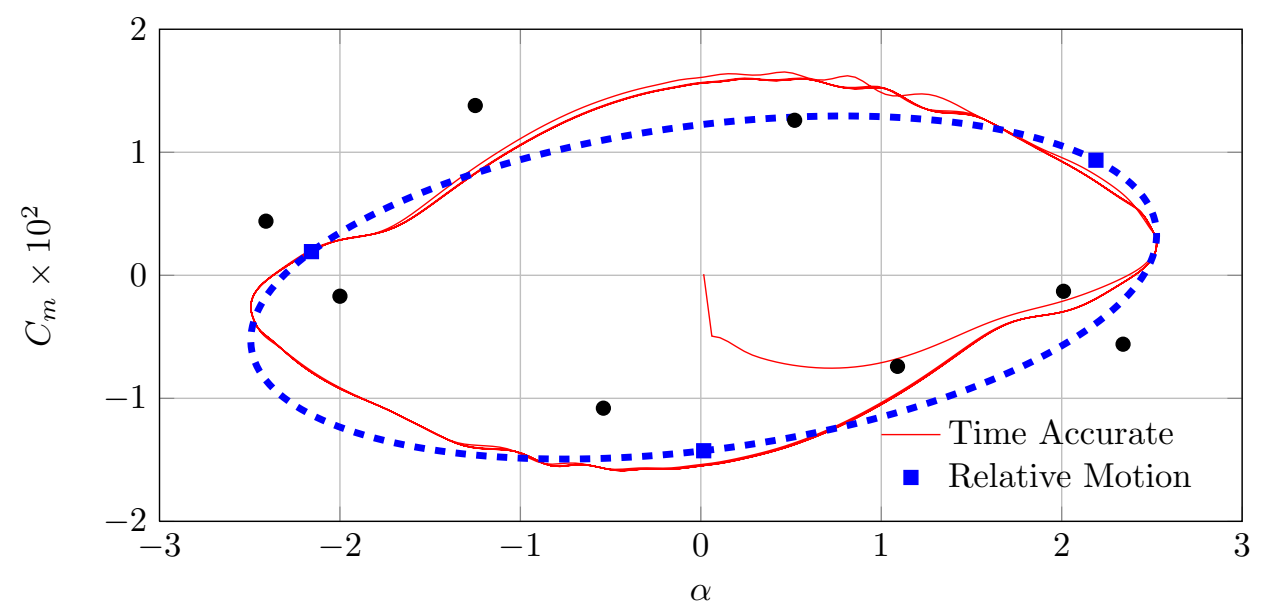

(a) $N=3$

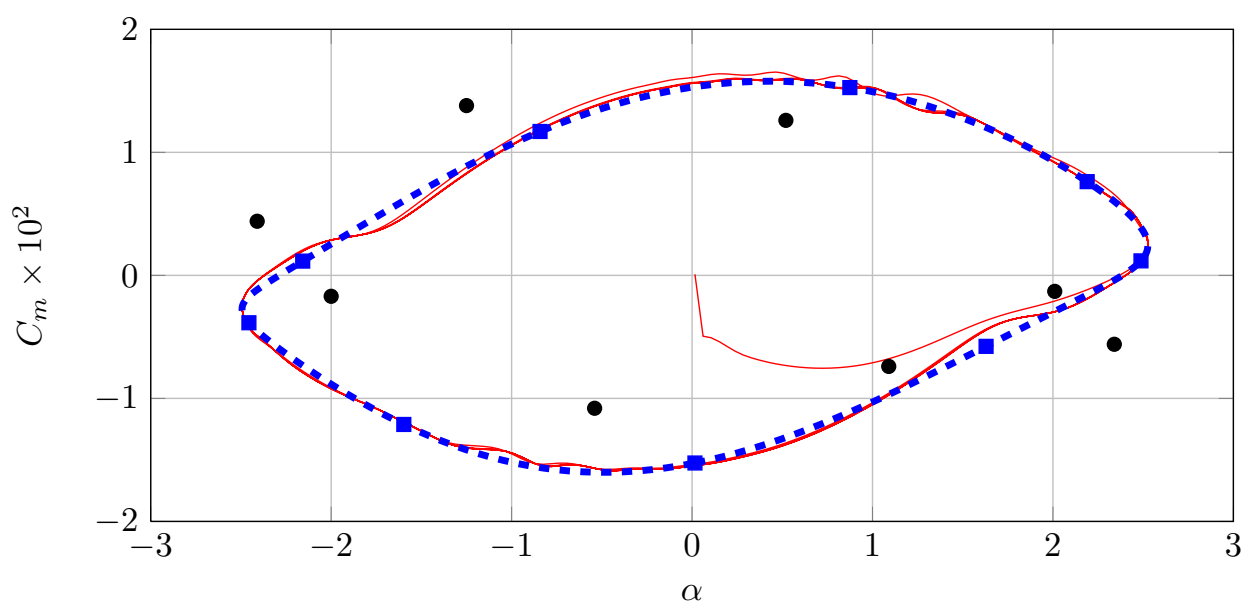

(b) $N=9$

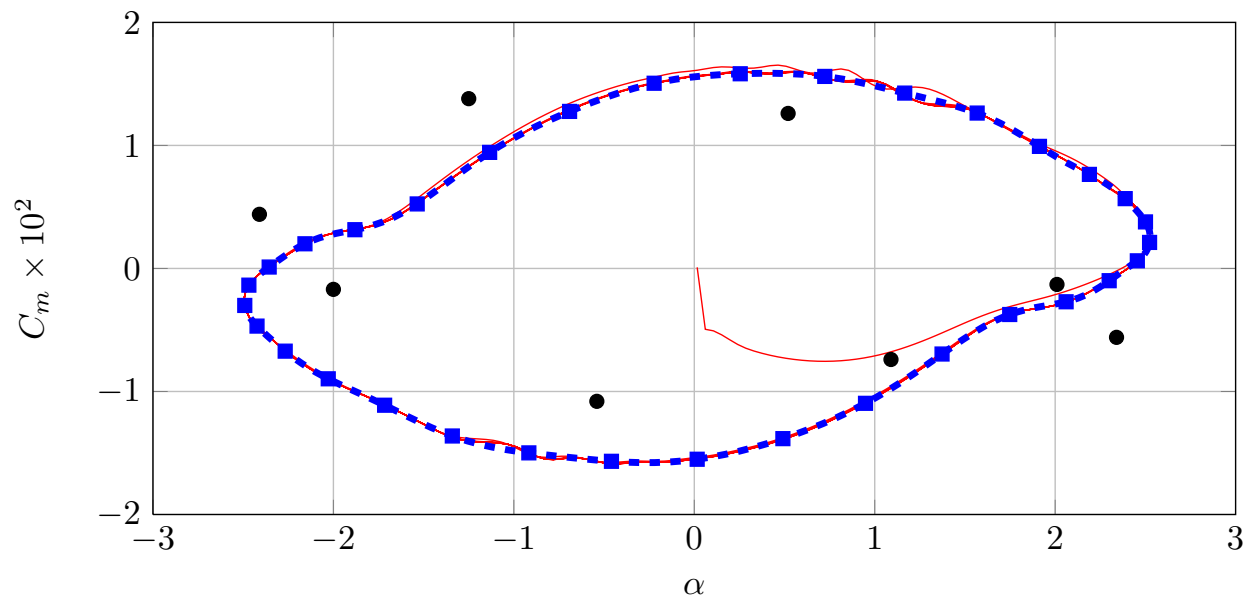

(c) $N=33$

Figure 20: AGARD 702 turbulent pitching airfoil. Time Spectral versus Time Accurate pitching moment coefficients for $N \in\{3,9,33\}$. Ten periods of the time-accurate solution are plotted in red from steady-state startup. Blue squares locate the pitching moment coefficient values at the Time-Spectral collocation points for relative-body motion. Relative-body pitching moment coefficients computed from an interpolation of the Time-Spectral solution to 201 points shown with the blue-hashed line. Experimental data from the AGARD 702 Report is plotted with black dots. 


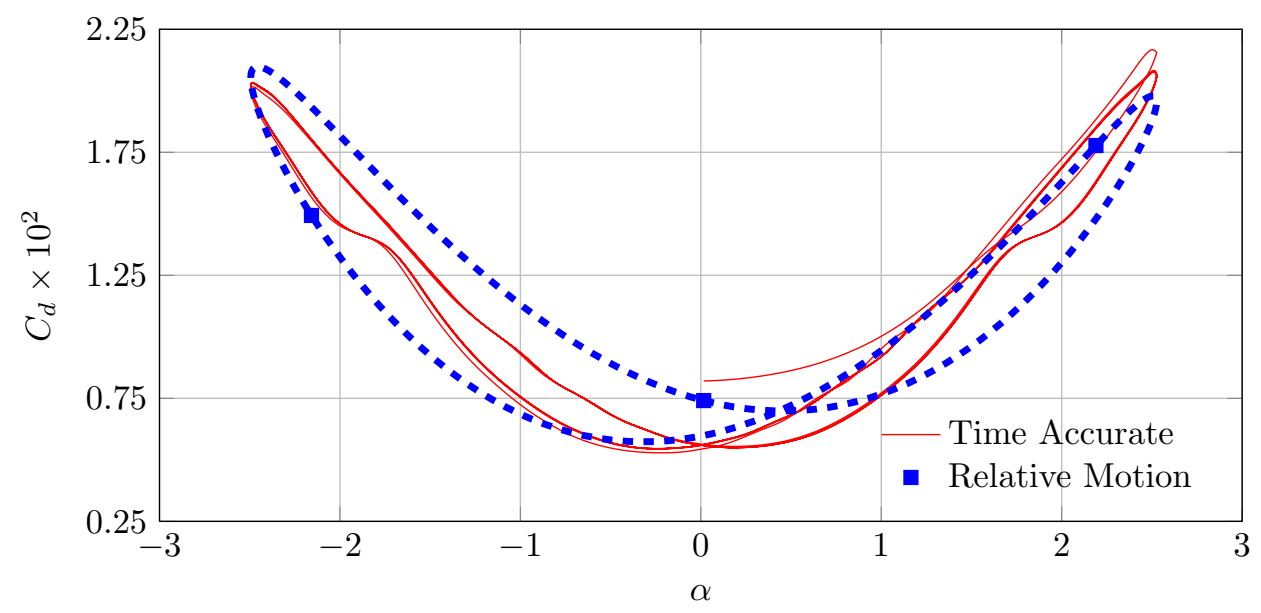

(a) $N=3$

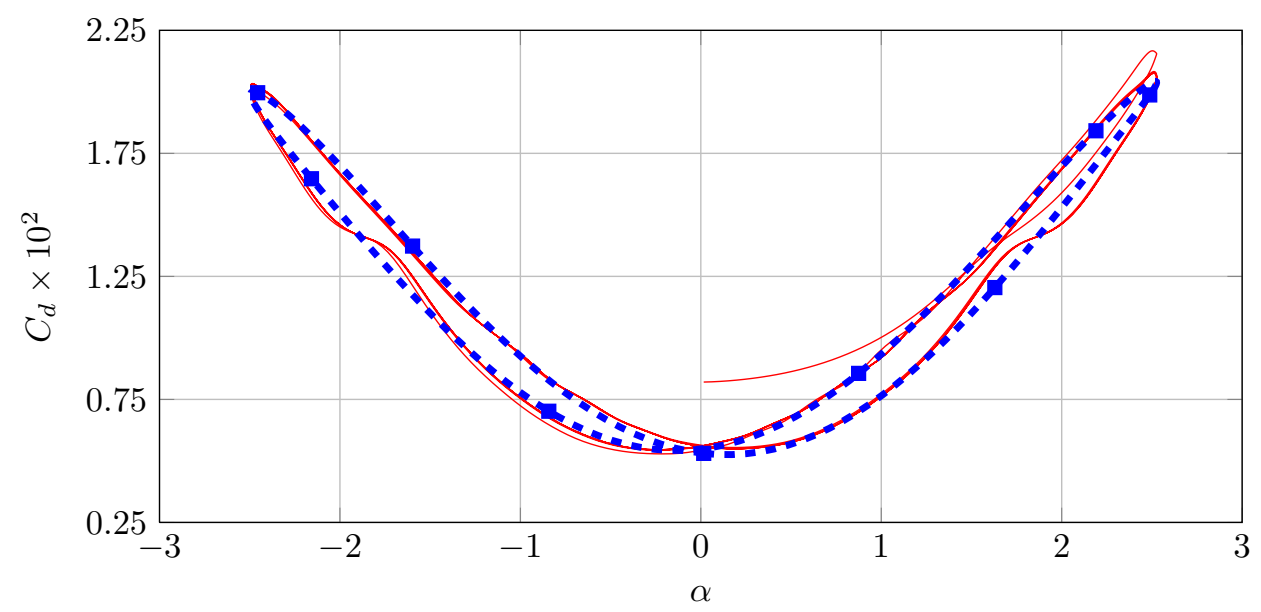

(b) $N=9$

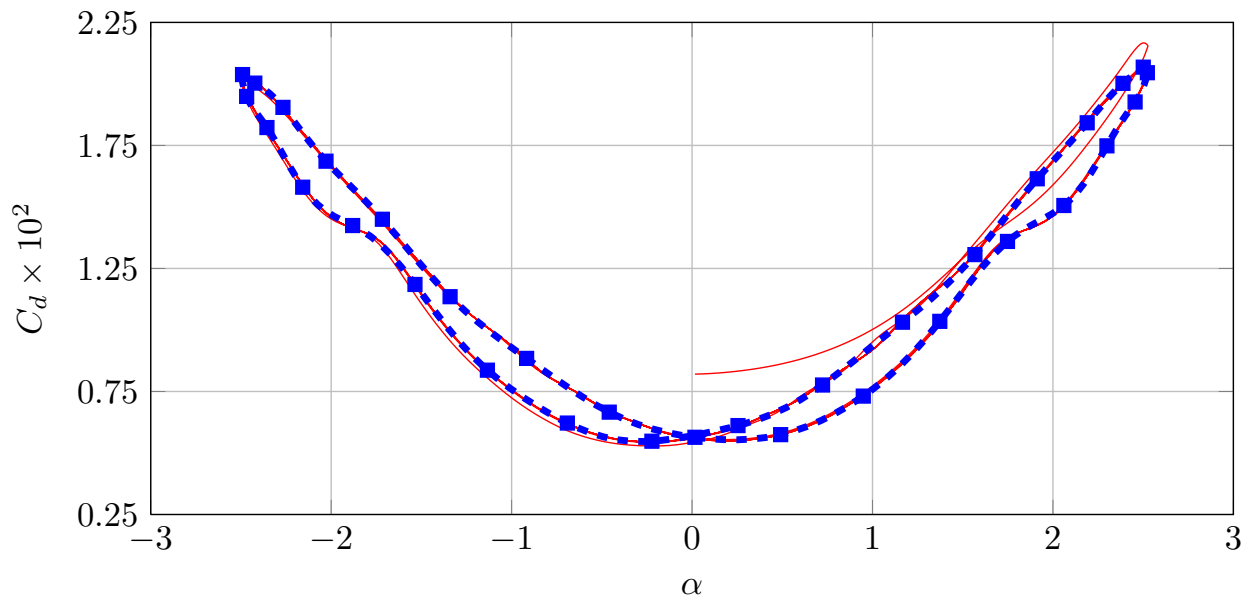

(c) $N=33$

Figure 21: AGARD 702 turbulent pitching airfoil. Time Spectral versus Time Accurate drag coefficients for $N \in\{3,9,33\}$. Ten periods of the time-accurate solution are plotted in red from steady-state startup. Blue squares locate the drag coefficient values at the Time-Spectral collocation points for relative-body motion. Relative-body drag coefficients computed from an interpolation of the Time-Spectral solution to 201 points shown with the blue-hashed line. Corrected experimental drag data from the AGARD 702 Report is not available. 


\section{Summary}

A new hybrid Time-Spectral method has been introduced for dynamic overset and Cartesian applications. The time samples of spatial nodes that undergo dynamic blanking are segmented into intervals of consecutively unblanked temporal collocation points and differentiated by an operator derived from barycentric rational polynomials. Barycentric rational polynomials offer superior interpolation and differentiation properties on an equispaced aperiodic mesh in contrast to traditional orthogonal polynomials. The majority of nodes are unblanked and treated by the standard Time-Spectral formulation.

The hybrid scheme was outlined and evaluated with a linear model problem and the implementation of the hybrid Time-Spectral scheme in the NASA OVERFLOW solver was described. Inviscid and turbulent test cases of plunging and pitching airfoils solved using both rigid and relative motion agreed with time-accurate solutions. The hybrid Time-Spectral scheme performed well in the inviscid subsonic plunging airfoil case where its conserved solution variables and drag coefficient values converged to the time-accurate data. The AGARD 702 inviscid pitching airfoil case demonstrated temporal convergence in its pitching moment and drag signals requiring $N=33$ modes to resolve the finest details of the solution. Spectrum analysis confirmed that no artificial high-frequency content was generated. A limiter was employed in the turbulent case to avoid large oscillations in the gradient of the turbulent eddy viscosity in the wake. Temporal convergence was similarly observed for the viscous case. The hybrid Time-Spectral scheme matched the results computed with both the conventional Time-Spectral approach and the time-accurate solver, establishing it as a viable tool for the simulation of periodically forced flows.

Future work includes acceleration of the code through multigrid and other techniques to enable application to more realistic problems in rotorcraft and turbomachinery. Dynamic near-body to near-body hole-cutting occurs in these problems and will therefore be investigated. Optimal selection of the rational polynomial approximation order, $d$, and its maximum value, $d_{\max }$, remains part of future work in addition to the stability analysis for the barycentric rational polynomial-based differentiation operator.

\section{References}

${ }^{1}$ Hall, K., Thomas, J., and Clark, W., "Computation of Unsteady Nonlinear Flows in Cascades using a Harmonic Balance Technique," 9th International Symposium on Unsteady Aerodynamics, Aeroacoustics and Aeroelasticity of Turbomachines, Lyon, France, September 2000.

${ }^{2}$ Hall, K., Thomas, J., and Clark, W., "Computation of Unsteady Nonlinear Flows in Cascades using a Harmonic Balance Technique," AIAA Journal, Vol. 40, 2002, pp. 879-886.

${ }^{3}$ McMullen, M. S. and Jameson, A., "The Computational Efficiency of Non-Linear Frequency Domain Methods," Journal of Computational Physics, Vol. 212, 2006, pp. 637-661.

${ }^{4}$ McMullen, M., Jameson, A., and Alonso, J., "Demonstration of Nonlinear Frequency Domain Methods," AIAA Journal, Vol. 44, No. 7, July 2006, pp. 1428-1435.

${ }^{5}$ Nadarajah, S. K., McMullen, M. S., and Jameson, A., "Optimum Shape Design for Unsteady Flow Using Time Accurate and Non-Linear Frequency Domain Methods," AIAA Paper 3875, June 2003.

${ }^{6}$ Murman, S. M., "A Reduced-Frequency Approach for Calculating Dynamic Derivatives," AIAA Journal, Vol. 45, No. 6, June 2007, pp. 1161-1168.

${ }^{7}$ Gopinath, A. K. and Jameson, A., "Time Spectral Method for Periodic Unsteady Computations over Two- and ThreeDimensional Bodies," AIAA Paper 2005-1220, January 2005.

${ }^{8}$ Gopinath, A. K. and Jameson, A., "Application of the Time Spectral Method to Periodic Vortex Shedding," AIAA Paper 2006-0449, January 2006.

${ }^{9}$ Blanc, F., Roux, F.-X., Jouhaud, J.-C., and Boussuge, J.-F., "Numerical Methods for Control Surfaces Aerodynamics with Flexibility Effects," IFASD, 2009.

${ }^{10}$ Choi, S., Potsdam, M., Lee, K., Iaccarino, G., and Alonso, J. J., "Helicopter Rotor Design Using a Time-Spectral and Adjoint-Based Method," AIAA Paper 5810, September 2008.

${ }^{11}$ Nadarajah, S. K. and Tatossian, C. A., "Adjoint-Based Aerodynamic Shape Optimization of Rotorcraft Blades," AIAA Paper 6730, August 2008.

${ }^{12}$ Mosahebi, A. and Nadarajah, S. K., "An Implicit Adaptive Non-Linear Frequency Domain Method (pNLFD) for Viscous Periodic Steady State Flows on Deformable Grids," AIAA Paper 0775, January 2011.

${ }^{13}$ Maple, R. C., King, P. I., Wolff, J. M., and Orkwis, P. D., "Split-Domain Harmonic Balance Solutions to Burger's Equation for Large-Amplitude Distrubances," AIAA Journal, Vol. 41, No. 2, February 2003, pp. 206-212.

${ }^{14}$ Maple, R. C., King, P. I., and Oxley, M. E., "Adaptive Harmonic Balance Solutions to Euler's Equation," AIAA Journal, Vol. 41, No. 9, September 2003, pp. 1705-1714.

${ }^{15}$ Thomas, J. P., Custer, C. H., Dowell, E. H., and Hall, K. C., "Unsteady Flow Computation Using a Harmonic Balance Approach Implemented about the OVERFLOW 2 Flow Solver," AIAA Paper 2009-4270, San Antonio, TX, June 2009.

${ }^{16}$ Custer, C. H., A Nonlinear Harmonic Balance Solver for an Implicit CFD Code: OVERFLOW 2, Ph.D. thesis, Duke University, 2009. 
${ }^{17}$ Mavriplis, D., Yang, Z., and Mundis, N., "Extensions of Time Spectral Methods for Practical Rotorcraft Problems," AIAA Paper 2012-0423, January 2012.

${ }^{18}$ Strang, G., "The Optimal Coefficients in Daubechies Wavelets," Physica D, Vol. 60, 1992, pp. 239-244.

${ }^{19}$ Romine, C. and Peyton, B., "Computing Connection Coefficients of Compactly Supported Wavelets on Bounded Intervals," Mathematical Sciences Section ORNL/TM-13413, Oak Ridge National Laboratory, April 1997.

${ }^{20}$ Huybrechs, D., "On the Fourier Extension of Nonperiodic Functions," SIAM Journal of Numerical Analysis, Vol. 47, No. 6, 2010, pp. 4326-4355.

${ }^{21}$ Boyd, J. P., "A Comparison of Numerical Algorithms for Fourier Extension of the First, Second, and Third Kinds," Journal of Computational Physics, Vol. 178, 2002, pp. 118-160.

${ }^{22}$ Bruno, O., Han, Y., and Pohlman, M. M., "Accurate, High-Order Representation of Complex Three-Dimensional Surfaces via Fourier Continuation Analysis," Journal of Computational Physics, Vol. 227, 2007, pp. 1094-1125.

${ }^{23}$ Candes, E., "Compressive Sampling," International Congress of Mathematicians, 2006.

${ }^{24}$ Platte, R. B., Trefethen, L. N., and Kuijlaars, A. B., "Impossibility of Fast Stable Approximations of Analytic Functions from Equispaced Samples," SIAM Review, Vol. 53, No. 2, 2011, pp. 308-318.

${ }^{25}$ Floater, M. S. and Hormann, K., "Barycentric Rational Interpolation with No Poles and High Rates of Approximation," Numerische Mathematik, Vol. 107, No. 2, August 2007, pp. 315-331.

${ }^{26}$ Bos, L., Marchi, S. D., Hormann, K., and Klein, G., "On the Lebesgue Constant of Barycentric Rational Interpolation at Equidistant Nodes," Journal of Computational and Applied Math, 2011.

${ }^{27}$ Baltensperger, R. and Berrut, J.-P., "The Linear Rational Collocation Method," Journal of Computational and Applied Math, Vol. 134, 2001, pp. 243-258.

${ }^{28}$ Berrut, J.-P. and Baltensperger, R., "The Linear Rational Pseudospectral Method for Boundary Value Problems," BIT, Vol. 41, No. 5, 2001, pp. 868-879.

${ }^{29}$ Klein, G., "An Extension of the Floater-Hormann Family of Barycentric Rational Interpolants," Mathematics of Computation, to appear, 2012.

${ }^{30}$ Tee, T. and Trefethen, L. N., "A Rational Spectral Collocation Method with Adaptively Transformed Chebyshev Grid Points," SIAM Journal of Scientific Computing, Vol. 28, No. 5, 2006, pp. 1798-1811.

${ }^{31}$ Baltensperger, R., Berrut, J.-P., and Noel, B., "Exponential Convergence of a Linear Rational Interpolant Between Transformed Chebyshev Points," Mathematics of Computation, Vol. 68, No. 227, 1999, pp. 1109-1120.

${ }^{32}$ Wang, Q., Moin, P., and Iaccarino, G., "A Rational Interpolation Scheme with Superpolynomial Rate of Convergence," SIAM Journal of Numerical Analysis, Vol. 47, No. 6, 2010, pp. 4073-4097.

${ }^{33}$ Nichols, R. and Buning, P., "Solver and Turbulence Model Upgrades to OVERFLOW 2 for Unsteady and High Speed Applications," AIAA Paper 2824, 2006.

${ }^{34}$ Landon, R., "Compendium of Unsteady Aerodynamic Measurements," AGARD Report No. 702, August 1982. 